Research in Astron. Astrophys. Vol.0 (200x) No.0, 000-000

http://www.raa-journal.org http://www.iop.org/journals/raa

Research in

Astronomy and

Astrophysics

\title{
Revisiting the Dichotomy of Early-type Galaxies
}

\author{
Yan-Qin $\mathrm{He}^{1,2,3}$, Cai-Na $\mathrm{Hao}^{3}$ and Xiao-Yang $\mathrm{Xia}^{3}$ \\ ${ }^{1}$ University of Chinese Academy of Sciences, Beijing 100049, China \\ 2 National Astronomical Observatories, Chinese Academy of Sciences, 20A Datun Road, Chaoyang \\ District, Beijing 100012, China \\ 3 Tianjin Astrophysics Center, Tianjin Normal University, Tianjin 300387, China \\ cainahao@gmail.com
}

\begin{abstract}
We study the relationship among isophotal shapes, central light profiles and kinematic properties of early-type galaxies (ETGs) based on a compiled sample of 184 ETGs. These sample galaxies are included in the Data Release 8 of Sloan Digital Sky Survey (SDSS DR8) and have central light profiles and kinematic properties available from the literature, which were measured based on Hubble Space Telescope (HST) and ATLAS $^{3 \mathrm{D}}$ integral-field spectrograph (IFS) observations, respectively. We find that there is only a weak correlation between the isophotal shape $\left(a_{4} / a\right)$ and the central light profile (within $1 \mathrm{kpc}$ ) of ETGs. About two-fifths of "core" galaxies have disky isophotes, while one-third of "power-law" galaxies are boxy deviated. Our statistical results also show that there are weak correlations between galaxy luminosity and dynamical mass with $a_{4} / a$, but such correlations are tighter with central light profile. Moreover, no clear link has been found between the isophotal shape and the Sersic index. Comparisons show that there are similar correlations between $a_{4} / a$ and ellipticity and between $a_{4} / a$ and specific angular momentum $\lambda_{R_{e} / 2}$ for "power-law" ETGs, but there are no such correlations for "core" ETGs. Therefore, we speculate that the bimodal classifications for ETGs are not as simple as previously thought, though we also find that the most deviated disky ETGs are "power-law", more elongated and fast rotators.
\end{abstract}

Key words: galaxies: elliptical and lenticular, $\mathrm{cD}$ — galaxies: kinematics and dynamics — galaxies: photometry — galaxies: structure

\section{INTRODUCTION}

The formation or assembly history of early-type galaxies (hereafter ETGs) has been a hot topic in the field of galaxy formation and evolution. Since the formation history of ETGs can imprint on their photometric and kinematical properties, extensive efforts have been made to explore these properties utilizing both imaging and spectroscopic observations. Since then significant progress has been made. Especially, dichotomies in isophotal shapes, nuclear light profiles and kinematics were found. In most cases, isophotal shapes are not perfect ellipses. Fourier analyses of the deviations from ellipses showed that the most significant non-zero component is the coefficient of the fourth cosine term (i.e., $a_{4} / a$; see Lauer 1985; Bender et al. 1988, 1989; Hao et al. 2006; Kormendy et al. 2009b). The sign of $a_{4} / a$ was used to divide ETGs into two classes: boxy $\left(a_{4} / a<0\right)$ and disky $\left(a_{4} / a>0\right)$ (Bender et al. 1988; Faber et al. 1997). Interestingly, some other properties also show that boxy and disky ETGs are two different populations. Boxy ETGs tend to be bright, have strong radio and X-ray emission, and rotate slowly while disky ETGs are faint, radio-quiet, have no X-ray hot gaseous halos and show regular rotation patterns. 
Similarly, dichotomy was also found in the central properties of ETGs. Based on high resolution images obtained by HST (Hubble Space Telescope), it was found that the central surface brightness profiles of ETGs could be fitted by a "Nuker Law" with the form of $\Sigma(\mathrm{r}) \sim \mathrm{r}^{-\gamma}$ (Crane et al. 1993; Ferrarese et al. 1994; Lauer et al. 1995). ETGs with steep inner cusps $(\gamma>0.5)$ are classified as "powerlaw" galaxies, while ETGs with shallow inner profiles $(\gamma<0.3)$ are called "core" galaxies (Lauer et al. 1995; Faber et al. 1997). In a more recent study, Lauer et al. (2007b) introduced $\gamma^{\prime}$ as an indicator of the bimodal classification, which is the local slope at the HST angular resolution limit, instead of $\gamma$ that is the inner cusp slope as $r \rightarrow 0$. ETGs with $\gamma^{\prime}>0.5$ are called "power-law" galaxies, "core" galaxies are those with $\gamma^{\prime}<0.3$, and the rest, i.e., ETGs with $0.3<\gamma^{\prime}<0.5$ are classified as "intermediate" type. The inner slope of the central profile of ETGs correlates with their global physical properties such as the luminosity, rotation velocity and isophotal shape (Faber et al. 1997; Lauer et al. 2007b).

The kinematics of ETGs were usually described by a ratio of the rotational velocity to the velocity dispersion $(v / \sigma)$. Recently, the SAURON Survey proposed a new tracer of the kinematical properties of ETGs, the specific angular momentum $\lambda_{R}$ (see section 3) to divide ETGs into fast and slow rotators (e.g. de Zeeuw et al. 2002; Emsellem et al. 2007). As an extension of the SAURON survey, the ATLAS $^{3 \mathrm{D}}$ team conducted a multi-wavelength survey for a carefully selected volume-limited ETGs sample with 260 objects using the SAURON integral-field spectrograph (IFS). Based on these observations, the ATLAS ${ }^{3 \mathrm{D}}$ team quantitatively classified ETGs into fast and slow rotators by using $\lambda_{R}=0.1$ (e.g. Krajnović et al. 2011). Fast rotators have regular stellar rotation with alignments between the photometric and kinematic axes, low luminosity and large ellipticity, while slow rotators show little or no rotation, and tend to be more massive and rather round (e.g. Cappellari et al. 2007; Emsellem et al. 2007, 2011).

Given that dichotomies have been found in isophotal shapes, nuclear light profiles and kinematics, it is interesting to investigate relations between these properties and with other galactic properties. Virtually, several studies have focused on such issues but reached conflicting conclusions. Krajnović et al. (2013) compared the nuclear light profiles and large scale kinematics of 135 ETGs, and concluded that there is no evidence for bimodal distribution in the nuclear slope. Emsellem et al. (2011) also pointed out that the $a_{4} / a$ parameter appears not to be directly related to the kinematic properties of ETGs. In a word, ATLAS ${ }^{3 D}$ team argues against the dichotomy of ETGs based on their isophotal shape $\left(a_{4} / a\right)$ and nuclear light profile. However, Lauer (2012) investigated the relation between the kinematics and central structures based on an ETG sample with 63 objects and found that they are well correlated if a criterion of $\lambda_{R_{e} / 2}=0.25$ (see section 3) is used to separate fast and slow rotators. The correlation shows that slow rotator ETGs usually have cores, while "power-law" galaxies tend to rotate rapidly.

From a theoretical point-of-view, simulations of galaxy formation indicate that the bimodality of isophotal shapes and central profiles of ETGs is correlated with galaxy merger histories, but such relations are complicated. The dissipationless simulations by Naab \& Burkert (2003) and Naab \& Trujillo (2006) showed that the equal-mass mergers of two disk galaxies tend to produce boxy ETGs, while unequal-mass mergers lead to disky ETGs. But Khochfar \& Burkert (2005) found that the isophotal shapes of merger remnants depend not only on the mass ratio of the last major merger, but also on the morphology of their progenitors and the subsequent gas infall. Using hydrodynamical simulations, Hopkins et al. (2009a, b) concluded that "power-law" ETGs are formed by dissipational mergers (wet-mergers) in the sense that the inner extra light/outer profile are formed in a compact central starburst/outer violent relaxation respectively, whereas "core" galaxies are formed by dry-mergers through subsequent merging of gas-poor ellipticals. During the process of dry-merging, the center becomes dense and compact because the merging binary black holes scatter out the inner stars.

Therefore, there is still a debate on the dichotomy of ETGs and their formation history from both observational and theoretical sides. In this work, we re-investigate the correlations among isophotal shapes, central light profiles and kinematic properties of ETGs based on a large compiled sample with 184 ETGs observed by both HST and SDSS DR8.

The paper is structured as follows. In section 2 we describe the sample used for this work. Then we outline the data reduction in section 3 . We present the main results in section 4 and finish with 
a summary in section 5. We adopt a Hubble constant of $\mathrm{H}_{0}=70 \mathrm{~km} \mathrm{~s}^{-1} \mathrm{Mpc}^{-1}$, a cosmology with matter density parameter $\Omega_{m}=0.3$, and a cosmological constant $\Omega_{\Lambda}=0.7$.

\section{SAMPLE}

To explore the relations between isophotal shapes, central light profiles and kinematics of ETGs, we need an ETG sample with these properties either available or measurable. As mentioned in the introduction, in the literature there are large ETGs samples observed with HST and their central light profiles have been carefully investigated. However, these observations only cover the central parts of the ETGs because of the small field of view of HST. Therefore SDSS images will be used to measure the global properties of ETGs instead.

Our ETGs sample is compiled from three sources. The first is from the cross-correlation of the SDSS DR8 photometric catalog with 219 ETGs collected by Lauer et al. (2007b). The sample galaxies in Lauer et al. (2007b) were observed by HST, WFPC2 $\left(36^{\prime \prime} .5 \times 36^{\prime \prime} .5,0^{\prime \prime} .046 /\right.$ pixel, Rest et al. 2001; Laine et al. 2003; Lauer et al. 2005), WFPC1 (66" $\times 66^{\prime \prime}, 0^{\prime \prime} .043 /$ pixel, Lauer et al. 1995; Faber et al. 1997) and NICMOS (19".2 $\times 19^{\prime \prime} .2,0^{\prime \prime} .076 /$ pixel, Quillen et al. 2000; Ravindranath et al. 2001). It encompasses 117 "core" galaxies, 89 "power-law" galaxies and 13 "intermediate" galaxies. The crosscorrelation of these 219 ETGs with the SDSS DR8 leads to 111 ETGs, in which there are 54 "core" galaxies, 54 "power-law" galaxies and 3 "intermediate" galaxies, respectively. The second is taken from Krajnović et al. (2013) with 135 ATLAS $^{3 \mathrm{D}}$ galaxies available in the HST archive. But 61 out of 135 ETGs have been included in Lauer et al. (2007b). A cross-correlation of the remaining 74 objects with the SDSS DR8 photometric catalog leaves us with 52 ETGs, consisting of 3 "core" galaxies, 37 "powerlaw" galaxies, and 12 "intermediate" galaxies. The third is from the cross-correlation of the SDSS DR8 photometric catalogue with the sample of 23 ETGs studied by Hyde et al. (2008), which were observed with Advanced Camera for Surveys (ACS) on HST and the velocity dispersions are larger than 350 $\mathrm{km} / \mathrm{s} .21$ ETGs were selected, including 6 "core" galaxies, 9 "power-law" galaxies and 6 "intermediate" galaxies. In total, we construct an ETGs sample with 184 galaxies, which consist of 63 "core" galaxies, 100 "power-law" galaxies and 21 "intermediate" galaxies, respectively. The redshifts of 111 ETGs from Lauer et al. (2007b) and 52 ETGs from Krajnović et al. (2013) are less than 0.04, while the 21 ETGs from Hyde et al. (2008) are in the range of $0.1<z<0.3$. All ETGs are in the luminosity range of $-24<M_{V}<-15$.

\section{DATA REDUCTION AND PARAMETER ESTIMATION}

We obtained the corrected frame fpC-images in the $r$-band for our sample ETGs directly from the SDSS DR8 Data Archive Server. For each frame $(2048 \times 1489$ pixels $)$, reductions including bias subtraction, flat-fielding, pixels defects and cosmic rays correction have been performed by the SDSS photometric pipeline (PHOTO, Lupton et al. 2001).

The background subtraction process is similar to that of Liu et al. (2008) and He et al. (2013), which has been successfully applied to the brightest ETGs. In the following, we outline this approach briefly. First, SExtractor (Bertin \& Amounts 1996) has been used to generate a background-only image with all detected objects flagged out. Then a median filter with $51 \times 51$ pixels is used to convolve the background-only image. After the median filtering, second-order Legendre polynomials are fitted to rows and columns separately by using the IRAF/NFIT1D task. Finally, we obtain the sky background model by using a circular Gaussian filter with $\sigma=9$ pixels to smooth the fitted frame. This sky background model is then subtracted from the original SDSS corrected frame. After sky background subtraction, the frame is trimmed to $501 \times 501$ pixels with the target galaxy centered and other objects masked out by using SExtractor. In the following, isophotal photometry will be performed on this final trimmed frame.

The IRAF/ELLIPSE task is used to perform the surface photometry. Given some initial guesses for galaxy geometric centre, ellipticity, semi-major axis length and position angle, the task fits the isophotes by a series of elliptical annuli from the centre to the outskirts, with a logarithmic step of 0.1 along 
the semi-major axis. The output of IRAF/ELLIPSE includes the mean isophotal intensity, the position angle P.A., the ellipticity $\epsilon$ for each annulus, and particularly the fourth harmonic deviations of Fourier analyses from the isophotal ellipses as a function of the semi-major axis. We derive the characteristic parameters $a_{4} / a$ and ellipticity $\epsilon$ by weighting them with the flux within the elliptical annulus over a region of twice FWHMs (full width of half-maximum) of seeing to the effective radius $R_{e}$.

Apart from $a_{4} / a$ and $\epsilon$, several other galactic properties including the Sérsic index, luminosity and dynamical mass are calculated. The Sérsic index $n$ and effective radius $R_{e}$ were obtained by fitting a point-spread function (PSF) convolved Sérsic model (Sérsic 1968) to the $r$-band sky-subtracted images using the algorithm GALFIT (Peng et al. 2002). The absolute magnitude is derived by $M=m-$ $5 \log \left(\mathrm{D}_{\mathrm{L}} / 10 \mathrm{pc}\right)-\mathrm{A}-\mathrm{k}$, where the apparent Petrosian magnitude $m$ and the extinction $A$ are taken from the SDSS DR8 photometric catalogue, $D_{\mathrm{L}}$ is the luminosity distance and the k-correction $k$ is derived using the KCORRECT algorithm of Blanton \& Roweis (2007). In order to transform the SDSS photometric data to the standard UBVRI Vega magnitude system, formula of Smith et al. (2002) has been used to get the absolute galaxy luminosities in the $V$-band. We derive the dynamical mass of sample ETGs based on the formula of $M_{d y n} \approx \sigma^{2} R_{e} / G$, where $\sigma$ is the corrected velocity dispersion at effective radius $R_{e}$ following von der Linden et al. (2007). In our sample ETGs, the velocity dispersions $\sigma$ are available for 136 objects, including the center velocity dispersions for 101 ETGs obtained from Lauer et al. (2007a) and the velocity dispersions of other 35 ETGs from the SDSS DR8 spectroscopic catalogue.

To compare with the central surface brightness profile and kinematic properties, the characteristic parameters $\gamma^{\prime}, r_{\gamma}$ and $\lambda_{R e / 2}$ were taken from the literature (Lauer et al. 2007a, b; Hyde et al. 2008; Cappellari et al. 2011; Krajnović et al. 2013). We describe briefly the way they were derived here. The central surface brightness profile is fitted by a "Nuker Law" with the following form

$$
I(r)=2^{(\beta-\gamma) / \alpha} I_{b}\left(\frac{r_{b}}{r}\right)^{\gamma}\left[1+\left(\frac{r}{r_{b}}\right)^{\alpha}\right]^{(\gamma-\beta) / \alpha},
$$

where the break radius $r_{b}$ is the point of maximum curvature in log-log coordinates, $I_{b}$ is the surface brightness at $r_{b},-\beta$ is the asymptotic outer slop, $\alpha$ is the sharpness of the break, and $\gamma$ is the inner cusp slope as $r \rightarrow 0$ and is distinguished from $\gamma^{\prime}$, which is the local slope evaluated at the HST angular resolution limit $r_{0}$, where

$$
\gamma^{\prime} \equiv-\left.\frac{d \log I}{d \log r}\right|_{r=r_{0}}=-\frac{\gamma+\beta\left(r_{0} / r_{b}\right)^{\alpha}}{1+\left(r_{0} / r_{b}\right)^{\alpha}} .
$$

As described in the introduction, the ETGs are classified into disky $\left(a_{4} / a>0\right)$ and boxy $\left(a_{4} / a<0\right)$ galaxies by their isophotal shapes, and they are also divided into "core" $\left(\gamma^{\prime}<0.3\right)$, "power-law" $\left(\gamma^{\prime}>\right.$ $0.5)$ and "intermediate" $\left(0.3<\gamma^{\prime}<0.5\right)$ galaxies according to their central light profiles. For "core" galaxies, the physical scale of the core is characterized by the "cusp radius", $r_{\gamma}$, which is a radius at which $\gamma^{\prime}$ equals 0.5 . Specifically, $r_{\gamma}$ is given by

$$
r_{\gamma} \equiv r_{b}\left(\frac{0.5-\gamma}{\beta-0.5}\right)^{1 / \alpha}
$$

The specific angular momentum parameter $\lambda_{R}$ is used as a discriminator of fast and slow rotators, where $\lambda_{R}$ is defined as

$$
\lambda_{R}=\frac{\sum_{n=1}^{N} F_{n} R_{n}\left|V_{n}\right|}{\sum_{n=1}^{N} F_{n} R_{n} \sqrt{V_{n}^{2}+\sigma_{n}^{2}}},
$$

where $F_{n}$ is the flux, $R_{n}$ is the circular radius from the center of the galaxy, $V_{n}$ and $\sigma_{n}$ are velocity and velocity dispersion inside the $n$-th spatial radial bin. Particularly, $\lambda_{R_{e} / 2}$ is the $\lambda_{R}$ measured within half of the effective radius $R_{e}$.

We list all these parameters for "core", "power-law" and "intermediate" galaxies in Tables 1,2 and 3. respectively. 
Table 1: "Core" Galaxy Parameters

\begin{tabular}{|c|c|c|c|c|c|c|c|c|c|c|c|}
\hline $\mathrm{N}$ & Galaxy & $\gamma^{\prime}$ & $\begin{array}{c}r_{\gamma} \\
\log (\mathrm{pc}) \\
(4)\end{array}$ & $\begin{array}{c}R_{e} \\
\log (\mathrm{pc}) \\
(5)\end{array}$ & $\begin{array}{c}\sigma \\
\mathrm{kms}_{(6)}^{-1} \\
(6)\end{array}$ & $\begin{array}{c}a_{4} / a \\
10^{(-2)} \\
(7) \\
\end{array}$ & $(8)$ & $\begin{array}{c}M_{V} \\
\operatorname{mag} \\
(9) \\
\end{array}$ & $\begin{array}{c}\log \frac{M_{d y n}}{M_{\odot}} \\
(10)\end{array}$ & $(11)$ & $\begin{array}{r}\lambda_{R_{e} / 2} \\
(12) \\
\end{array}$ \\
\hline 1 & IC 0613 & $0.25^{b}$ & $2.05^{a}$ & 3.97 & $262^{a}$ & $0.125 \pm 0.010$ & $0.084 \pm 0.002$ & -22.27 & 11.17 & 5.26 & - \\
\hline 2 & IC 0664 & $0.12^{b}$ & $2.07^{a}$ & 4.84 & $336^{a}$ & $-0.286 \pm 0.014$ & $0.229 \pm 0.002$ & -22.86 & 12.26 & 8.87 & - \\
\hline 3 & IC 0712 & $0.17^{b}$ & $2.69^{a}$ & 4.75 & $345^{a}$ & $0.132 \pm 0.006$ & $0.188 \pm 0.001$ & -23.29 & 12.19 & 7.79 & - \\
\hline 4 & IC 1565 & $-0.03^{b}$ & $1.65^{a}$ & 3.75 & $303^{a}$ & $-0.008 \pm 0.074$ & $0.046 \pm 0.002$ & -22.99 & 11.08 & 14.59 & - \\
\hline 5 & IC 1695 & $0.23^{b}$ & $2.36^{a}$ & 4.74 & $364^{a}$ & $0.049 \pm 0.097$ & $0.234 \pm 0.004$ & -23.90 & 12.23 & 8.22 & - \\
\hline 6 & IC 1733 & $-0.01^{b}$ & $2.68^{a}$ & 4.59 & $301^{a}$ & $0.433 \pm 0.088$ & $0.126 \pm 0.002$ & -23.43 & 11.91 & 4.87 & - \\
\hline 7 & J010803.2+151333.6 & $0.23^{d}$ & - & 4.22 & $304^{e}$ & $-0.026 \pm 0.054$ & $0.170 \pm 0.007$ & -23.19 & 11.55 & 3.28 & - \\
\hline 8 & J083445.2+355142.0 & $0.06^{d}$ & - & 4.60 & $366^{e}$ & $0.067 \pm 0.088$ & $0.175 \pm 0.007$ & -23.71 & 12.09 & 4.96 & - \\
\hline 9 & J124609.4+515021.6 & $0.21^{d}$ & - & 4.38 & $387^{e}$ & $0.509 \pm 0.090$ & $0.097 \pm 0.012$ & -23.85 & 11.92 & 3.99 & - \\
\hline 10 & $\mathrm{~J} 141341.4+033104.3$ & $-0.09^{d}$ & - & 4.77 & $364^{e}$ & $-0.119 \pm 0.112$ & $0.162 \pm 0.012$ & -23.42 & 12.26 & 1.89 & - \\
\hline 11 & J171328.4+274336.6 & $0.04^{d}$ & - & 4.49 & $414^{e}$ & $0.865 \pm 0.093$ & $0.171 \pm 0.013$ & -24.17 & 12.09 & 5.61 & - \\
\hline 12 & J211019.2+095047.1 & $0.17^{d}$ & - & 4.25 & $371^{e}$ & $-0.981 \pm 0.053$ & $0.144 \pm 0.006$ & -23.78 & 11.76 & 2.00 & - \\
\hline 13 & MCG 11-14-25A & $0.30^{b}$ & $1.38^{a}$ & 3.34 & $148^{e}$ & $0.185 \pm 0.007$ & $0.097 \pm 0.001$ & -19.08 & 10.05 & 4.34 & - \\
\hline 14 & NGC 0524 & $0.27^{b}$ & $1.57^{a}$ & 3.50 & $253^{a}$ & $0.218 \pm 0.035$ & $0.034 \pm 0.001$ & -21.85 & 10.67 & 4.55 & 0.325 \\
\hline 15 & NGC 0545 & $0.10^{b}$ & $2.16^{a}$ & 4.36 & $242^{a}$ & $0.462 \pm 0.059$ & $0.239 \pm 0.001$ & -22.98 & 11.49 & 16.99 & - \\
\hline 16 & NGC 0584 & $0.30^{b}$ & $0.95^{a}$ & 3.53 & $207^{a}$ & $0.106 \pm 0.028$ & $0.250 \pm 0.001$ & -21.38 & 10.53 & 7.06 & - \\
\hline 17 & NGC 0741 & $0.11^{b}$ & $2.46^{a}$ & 4.12 & $291^{a}$ & $0.090 \pm 0.037$ & $0.128 \pm 0.001$ & -23.27 & 11.41 & 6.18 & - \\
\hline 18 & NGC 1016 & $0.11^{b}$ & $2.25^{a}$ & 2.10 & $294^{a}$ & $-0.026 \pm 0.035$ & $0.066 \pm 0.001$ & -22.90 & 9.40 & 7.74 & - \\
\hline 19 & NGC 1052 & $0.22^{b}$ & $1.46^{a}$ & 3.45 & $208^{a}$ & $-0.773 \pm 0.001$ & $0.265 \pm 0.001$ & -21.17 & 10.45 & 2.75 & - \\
\hline 20 & NGC 1700 & $0.07^{b}$ & $1.01^{a}$ & 3.64 & $235^{a}$ & $0.986 \pm 0.029$ & $0.266 \pm 0.001$ & -21.95 & 10.75 & 12.12 & - \\
\hline 21 & NGC 2832 & $0.03^{b}$ & $2.52^{a}$ & 4.93 & $335^{a}$ & $-0.333 \pm 0.003$ & $0.192 \pm 0.001$ & -23.76 & 12.35 & 9.08 & - \\
\hline 22 & NGC 3193 & $0.28^{b}$ & $1.38^{a}$ & 3.49 & $194^{a}$ & $0.317 \pm 0.001$ & $0.161 \pm 0.001$ & -21.98 & 10.43 & 4.92 & 0.197 \\
\hline 23 & NGC 3379 & $0.18^{b}$ & $1.72^{a}$ & 3.67 & $207^{a}$ & $-0.028 \pm 0.001$ & $0.098 \pm 0.001$ & -21.14 & 10.67 & 6.66 & 0.157 \\
\hline 24 & NGC 3551 & $0.14^{b}$ & $2.37^{a}$ & 5.44 & $268^{a}$ & $0.374 \pm 0.005$ & $0.173 \pm 0.001$ & -23.55 & 12.66 & 9.43 & - \\
\hline 25 & NGC 3607 & $0.26^{b}$ & $1.77^{a}$ & 3.51 & $224^{a}$ & $-0.099 \pm 0.001$ & $0.192 \pm 0.001$ & -19.88 & 10.58 & 4.61 & 0.228 \\
\hline 26 & NGC 3608 & $0.17^{b}$ & $1.31^{a}$ & 3.73 & $193^{a}$ & $-0.420 \pm 0.001$ & $0.175 \pm 0.001$ & -21.12 & 10.67 & 5.71 & 0.043 \\
\hline 27 & NGC 3613 & $0.08^{b}$ & $1.65^{a}$ & 3.56 & $210^{a}$ & $-0.123 \pm 0.003$ & $0.313 \pm 0.001$ & -21.59 & 10.57 & 2.92 & 0.191 \\
\hline 28 & NGC 3640 & $0.03^{b}$ & $1.47^{a}$ & 3.41 & $182^{a}$ & $-0.305 \pm 0.001$ & $0.214 \pm 0.001$ & -21.96 & 10.30 & 3.41 & 0.320 \\
\hline 29 & NGC 3842 & $0.12^{b}$ & $2.48^{a}$ & 4.41 & $314^{a}$ & $-0.387 \pm 0.003$ & $0.149 \pm 0.001$ & -23.18 & 11.77 & 5.59 & - \\
\hline 30 & NGC 4073 & $-0.08^{b}$ & $2.13^{a}$ & 4.56 & $278^{a}$ & $0.349 \pm 0.003$ & $0.297 \pm 0.001$ & -23.50 & 11.81 & 5.16 & - \\
\hline 31 & NGC 4168 & $0.17^{b}$ & $2.26^{a}$ & 3.76 & $184^{a}$ & $0.804 \pm 0.002$ & $0.155 \pm 0.001$ & -21.80 & 10.66 & 3.61 & 0.040 \\
\hline 32 & NGC 4261 & $0.00^{b}$ & $2.31^{a}$ & 3.96 & $309^{a}$ & $-1.372 \pm 0.001$ & $0.256 \pm 0.001$ & -22.26 & 11.31 & 5.31 & 0.085 \\
\hline 33 & NGC 4278 & $0.10^{b}$ & $1.77^{a}$ & 3.16 & $238^{a}$ & $-0.280 \pm 0.001$ & $0.148 \pm 0.001$ & -21.05 & 10.28 & 4.49 & 0.203 \\
\hline 34 & NGC 4365 & $0.09^{b}$ & $2.15^{a}$ & 4.06 & $256^{a}$ & $-1.181 \pm 0.001$ & $0.238 \pm 0.001$ & -22.18 & 11.24 & 6.26 & 0.088 \\
\hline 35 & NGC 4371 & $0.27^{c}$ & $1.60^{c}$ & 3.24 & - & $0.512 \pm 0.061$ & $0.257 \pm 0.002$ & -20.00 & - & 3.43 & 0.482 \\
\hline 36 & NGC 4374 & $0.13^{b}$ & $2.11^{a}$ & 3.79 & $282^{a}$ & $-0.401 \pm 0.001$ & $0.183 \pm 0.002$ & -22.28 & 11.06 & 5.62 & 0.024 \\
\hline 37 & NGC 4382 & $0.01^{b}$ & $1.69^{a}$ & 4.04 & $179^{a}$ & $0.852 \pm 0.001$ & $0.212 \pm 0.001$ & -21.96 & 10.91 & 6.00 & 0.163 \\
\hline 38 & NGC 4406 & $-0.04^{b}$ & $1.90^{a}$ & 3.34 & $235^{a}$ & $-0.763 \pm 0.001$ & $0.180 \pm 0.001$ & -22.46 & 10.45 & 6.68 & 0.052 \\
\hline 39 & NGC 4458 & $0.17^{b}$ & $0.80^{a}$ & 3.51 & $103^{a}$ & $0.395 \pm 0.003$ & $0.138 \pm 0.001$ & -19.27 & 9.90 & 6.97 & 0.079 \\
\hline 40 & NGC 4472 & $0.01^{b}$ & $2.25^{a}$ & 3.56 & $291^{a}$ & $-0.227 \pm 0.001$ & $0.087 \pm 0.001$ & -22.93 & 10.85 & 3.01 & 0.077 \\
\hline 41 & NGC 4473 & $0.01^{b}$ & $1.73^{a}$ & 3.67 & $179^{a}$ & $1.149 \pm 0.001$ & $0.388 \pm 0.001$ & -21.16 & 10.54 & 4.28 & 0.250 \\
\hline 42 & NGC 4478 & $0.10^{b}$ & $1.32^{a}$ & 3.08 & $138^{a}$ & $-0.449 \pm 0.002$ & $0.181 \pm 0.001$ & -19.89 & 9.73 & 1.84 & 0.177 \\
\hline 43 & NGC 4486 & $0.27^{b}$ & $2.65^{a}$ & 3.53 & $332^{a}$ & $-0.098 \pm 0.001$ & $0.017 \pm 0.001$ & -22.71 & 10.94 & 2.14 & - \\
\hline 44 & NGC 4486B & $-0.10^{b}$ & $1.08^{a}$ & 2.49 & $170^{a}$ & $0.458 \pm 0.005$ & $0.110 \pm 0.002$ & -17.98 & 9.32 & 2.10 & 0.021 \\
\hline 45 & NGC 4552 & $-0.02^{b}$ & $1.60^{a}$ & 2.89 & $253^{a}$ & $-0.010 \pm 0.001$ & $0.050 \pm 0.001$ & -21.65 & 10.06 & 4.43 & 0.049 \\
\hline 46 & NGC 4636 & $0.13^{b}$ & $2.21^{a}$ & 3.59 & $203^{a}$ & $-0.018 \pm 0.001$ & $0.026 \pm 0.001$ & -21.86 & 10.57 & 3.44 & 0.036 \\
\hline 47 & NGC 4649 & $0.17^{b}$ & $2.34^{a}$ & 3.62 & $336^{a}$ & $-0.477 \pm 0.001$ & $0.113 \pm 0.001$ & -22.51 & 11.04 & 3.23 & 0.127 \\
\hline 48 & NGC 4874 & $0.12^{b}$ & $2.99^{a}$ & 4.80 & $278^{a}$ & $-0.058 \pm 0.003$ & $0.074 \pm 0.001$ & -23.49 & 12.05 & 4.96 & - \\
\hline 49 & NGC 4889 & $0.03^{b}$ & $2.84^{a}$ & 4.11 & $401^{a}$ & $-0.563 \pm 0.002$ & $0.268 \pm 0.001$ & -23.73 & 11.68 & 3.41 & - \\
\hline 50 & NGC 5198 & $0.26^{b}$ & $1.33^{a}$ & 3.60 & $196^{a}$ & $-0.249 \pm 0.003$ & $0.130 \pm 0.001$ & -21.23 & 10.55 & 3.53 & 0.057 \\
\hline
\end{tabular}


He et al.

\begin{tabular}{|c|c|c|c|c|c|c|c|c|c|c|c|}
\hline 51 & NGC 5322 & $0.15^{c}$ & $2.02^{c}$ & 3.64 & - & $-0.001 \pm 0.029$ & $0.320 \pm 0.001$ & -21.41 & - & 6.11 & 0.067 \\
\hline 52 & NGC 5485 & $0.19^{c}$ & $1.90^{c}$ & 3.75 & $176^{e}$ & $-0.599 \pm 0.450$ & $0.068 \pm 0.010$ & -21.14 & 10.61 & 5.09 & 0.149 \\
\hline 53 & NGC 5557 & $0.07^{b}$ & $1.82^{a}$ & 3.99 & $254^{a}$ & $-0.274 \pm 0.002$ & $0.202 \pm 0.001$ & -22.62 & 11.17 & 5.33 & 0.045 \\
\hline 54 & NGC 5576 & $0.26^{b}$ & $1.21^{a}$ & 3.36 & $183^{a}$ & $-0.642 \pm 0.001$ & $0.258 \pm 0.001$ & -21.31 & 10.25 & 4.65 & 0.091 \\
\hline 55 & NGC 5813 & $0.06^{b}$ & $1.89^{a}$ & 4.72 & $239^{a}$ & $0.042 \pm 0.001$ & $0.095 \pm 0.001$ & -22.01 & 11.84 & 8.50 & 0.071 \\
\hline 56 & NGC 5982 & $0.05^{b}$ & $1.80^{a}$ & 3.81 & $240^{a}$ & $-1.241 \pm 0.002$ & $0.281 \pm 0.001$ & -21.97 & 10.94 & 4.92 & - \\
\hline 57 & NGC 6086 & $0.02^{b}$ & $2.53^{a}$ & 4.57 & $336^{a}$ & $-0.562 \pm 0.005$ & $0.268 \pm 0.001$ & -23.11 & 11.99 & 7.36 & - \\
\hline 58 & NGC 6166 & $0.12^{b}$ & $3.17^{a}$ & 4.40 & $310^{a}$ & $-0.372 \pm 0.005$ & $0.203 \pm 0.002$ & -23.80 & 11.75 & 2.75 & - \\
\hline 59 & NGC 6173 & $0.02^{b}$ & $2.32^{a}$ & 4.60 & $278^{a}$ & $-0.334 \pm 0.004$ & $0.332 \pm 0.001$ & -23.59 & 11.85 & 6.87 & - \\
\hline 60 & NGC $7578 B$ & $0.21^{b}$ & $2.06^{a}$ & 4.57 & $214^{a}$ & $0.499 \pm 0.085$ & $0.170 \pm 0.002$ & -23.41 & 11.60 & 16.47 & - \\
\hline 61 & NGC 7619 & $0.01^{b}$ & $2.03^{a}$ & 3.97 & $322^{a}$ & $0.238 \pm 0.036$ & $0.231 \pm 0.001$ & -22.94 & 11.35 & 6.25 & - \\
\hline 62 & NGC 7647 & $0.05^{b}$ & $2.28^{a}$ & 3.00 & $282^{a}$ & $0.994 \pm 0.294$ & $0.290 \pm 0.035$ & -23.97 & 10.27 & 15.58 & - \\
\hline 63 & NGC 7785 & $0.06^{b}$ & $1.32^{a}$ & 3.62 & $245^{a}$ & $-1.708 \pm 0.040$ & $0.388 \pm 0.001$ & -22.08 & 10.76 & 4.84 & - \\
\hline
\end{tabular}

Notes: Col.(1): Number. Col.(2): Galaxy Name. Cols.(3) and (4): The local slope of "Nuker Law" fits and "Cusp radius", ${ }^{a}$ from Lauer et al. (2007a); ${ }^{b}$ from Lauer et al. (2007b); ${ }^{c}$ from Krajnović et al. (2013); ${ }^{d}$ from Hyde et al. (2008). Col.(5): Effective radius from best Sérsic fits. Col.(6): Central velocity dispersion, ${ }^{d}$ from Hyde et al. (2008); ${ }^{e}$ from the SDSS DR8 Spectroscopic catalogue. Col.(7): Isophotal shape parameter $a_{4} / a$. Col.(8): Ellipticity. Col.(9): Absolute magnitude in the $V$-band. Col.(10): The dynamical mass. Col.(11): Sérsic index. Col.(12): Specific angular momentum parameter from Emsellem et al. (2011).

Table 2: "Power-law" Galaxy Parameters

\begin{tabular}{|c|c|c|c|c|c|c|c|c|c|c|c|}
\hline (1) & Galaxy & $\gamma^{\prime}$ & $\begin{array}{c}r_{\gamma} \\
\log (p c) \\
(4)\end{array}$ & $\begin{array}{c}R_{e} \\
\log (\mathrm{pc}) \\
(5)\end{array}$ & $\begin{array}{c}\sigma \\
\mathrm{km} \mathrm{s}^{-1} \\
(6)\end{array}$ & $\begin{array}{c}a_{4} / a \\
10^{(-2)} \\
(7) \\
\end{array}$ & (8) & $\begin{array}{c}M_{V} \\
\operatorname{mag} \\
(9) \\
\end{array}$ & $\begin{array}{c}\log \frac{M_{d y n}}{M_{\odot}} \\
(10)\end{array}$ & $(11)$ & $\lambda_{R_{e} / 2}$ \\
\hline 1 & IC 0875 & $1.12^{b}$ & $1.01^{a}$ & 4.27 & - & $0.679 \pm 0.006$ & $0.394 \pm 0.001$ & -20.21 & - & 11.20 & - \\
\hline 2 & IC 2738 & $0.60^{b}$ & $1.57^{a}$ & 4.04 & $275^{a}$ & $0.202 \pm 0.009$ & $0.068 \pm 0.002$ & -22.18 & 11.29 & 5.84 & - \\
\hline 3 & J013431.5+131436.4 & $0.54^{d}$ & - & 4.17 & $248^{e}$ & $-0.214 \pm 0.309$ & $0.524 \pm 0.024$ & -23.17 & 11.33 & 9.65 & - \\
\hline 4 & J082216.5+481519.1 & $0.94^{d}$ & - & 5.90 & $351^{e}$ & $-0.170 \pm 0.155$ & $0.305 \pm 0.012$ & -21.26 & 13.36 & 15.00 & - \\
\hline 5 & J082646.7+495211.5 & $1.14^{d}$ & - & 4.47 & - & $0.113 \pm 0.160$ & $0.305 \pm 0.014$ & -22.10 & - & 4.83 & - \\
\hline 6 & J093124.4+574926.6 & $0.52^{d}$ & - & 4.17 & $350^{e}$ & $0.988 \pm 0.117$ & $0.235 \pm 0.012$ & -22.95 & 11.62 & 2.20 & - \\
\hline 7 & $\mathrm{~J} 103344.2+043143.5$ & $0.80^{d}$ & - & 4.12 & $335^{e}$ & $-0.258 \pm 0.117$ & $0.414 \pm 0.010$ & -22.36 & 11.54 & 3.36 & - \\
\hline 8 & $\mathrm{~J} 111525.7+024033.9$ & $0.76^{d}$ & - & 4.34 & $379^{e}$ & $-0.833 \pm 0.108$ & $0.265 \pm 0.011$ & -23.40 & 11.86 & 3.21 & - \\
\hline 9 & J151741.7-004217.6 & $1.10^{d}$ & - & 3.93 & $380^{e}$ & $0.115 \pm 0.059$ & $0.215 \pm 0.005$ & -21.67 & 11.46 & 3.10 & - \\
\hline 10 & $\mathrm{~J} 160239.1+022110.0$ & $0.61^{d}$ & - & 4.00 & $358^{e}$ & $1.654 \pm 0.198$ & $0.271 \pm 0.017$ & -22.91 & 11.47 & 3.08 & - \\
\hline 11 & $\mathrm{~J} 221414.3+131703.7$ & $1.09^{d}$ & - & 3.60 & - & $1.689 \pm 0.094$ & $0.274 \pm 0.009$ & -21.85 & - & 2.18 & - \\
\hline 12 & MCG 08-27-18 & $0.89^{b}$ & $1.07^{a}$ & 3.18 & $89^{a}$ & $-0.074 \pm 0.006$ & $0.076 \pm 0.001$ & -20.03 & 9.45 & 3.41 & - \\
\hline 13 & NGC 0474 & $0.56^{b}$ & $1.15^{a}$ & 3.59 & $164^{a}$ & $-0.212 \pm 0.043$ & $0.122 \pm 0.001$ & -20.12 & 10.39 & 9.24 & 0.210 \\
\hline 14 & NGC 0596 & $0.54^{b}$ & $0.63^{a}$ & 3.51 & $152^{a}$ & $0.034 \pm 0.029$ & $0.069 \pm 0.001$ & -20.90 & 10.24 & 8.28 & - \\
\hline 15 & NGC 0936 & $0.52^{c}$ & $0.87^{c}$ & 3.50 & - & $0.145 \pm 0.025$ & $0.108 \pm 0.001$ & -20.84 & - & 6.13 & 0.430 \\
\hline 16 & NGC 2549 & $0.67^{b}$ & $0.51^{a}$ & 3.25 & $143^{a}$ & $1.938 \pm 0.003$ & $0.447 \pm 0.001$ & -19.17 & 9.93 & 3.18 & 0.523 \\
\hline 17 & NGC 2592 & $0.92^{b}$ & $0.82^{a}$ & 3.24 & $265^{a}$ & $0.579 \pm 0.003$ & $0.158 \pm 0.001$ & -20.01 & 10.45 & 3.53 & 0.431 \\
\hline 18 & NGC 2685 & $0.73^{b}$ & $0.84^{a}$ & 3.23 & $94^{a}$ & $3.180 \pm 0.004$ & $0.533 \pm 0.002$ & -19.72 & 9.54 & 3.59 & 0.632 \\
\hline 19 & NGC 2778 & $0.83^{b}$ & $0.67^{a}$ & 3.31 & $162^{a}$ & $0.721 \pm 0.004$ & $0.208 \pm 0.001$ & -18.75 & 10.10 & 1.86 & 0.435 \\
\hline 20 & NGC 2859 & $0.76^{c}$ & $0.77^{c}$ & 3.18 & - & $1.109 \pm 0.035$ & $0.188 \pm 0.001$ & -20.83 & - & 3.60 & 0.361 \\
\hline 21 & NGC 2872 & $1.01^{b}$ & $1.06^{a}$ & 3.65 & $285^{a}$ & $-0.147 \pm 0.002$ & $0.200 \pm 0.001$ & -21.62 & 10.93 & 4.15 & - \\
\hline 22 & NGC 2880 & $0.75^{c}$ & $1.01^{c}$ & 3.29 & $281^{e}$ & $-0.215 \pm 0.035$ & $0.210 \pm 0.001$ & -20.31 & 10.56 & 4.95 & 0.482 \\
\hline 23 & NGC 2950 & $0.82^{b}$ & $0.58^{a}$ & 3.27 & $182^{a}$ & $0.819 \pm 0.002$ & $0.242 \pm 0.001$ & -19.73 & 10.16 & 5.17 & 0.428 \\
\hline 24 & NGC 2962 & $0.80^{c}$ & $1.21^{c}$ & 3.77 & - & $1.509 \pm 0.076$ & $0.280 \pm 0.001$ & -20.42 & - & 8.37 & 0.329 \\
\hline 25 & NGC 3156 & $1.78^{c}$ & $1.02^{c}$ & 3.85 & - & $-0.252 \pm 0.062$ & $0.435 \pm 0.001$ & -19.36 & - & 8.62 & 0.559 \\
\hline 26 & NGC 3226 & $0.83^{c}$ & $0.57^{c}$ & 3.87 & - & $-0.267 \pm 0.048$ & $0.162 \pm 0.001$ & -19.59 & - & 9.59 & 0.257 \\
\hline 27 & NGC 3245 & $0.74^{c}$ & $0.99^{c}$ & 3.63 & $850^{e}$ & $0.476 \pm 0.032$ & $0.318 \pm 0.001$ & -20.62 & 11.86 & 8.60 & 0.592 \\
\hline 28 & NGC 3266 & $0.66^{b}$ & $0.85^{a}$ & 3.40 & - & $1.889 \pm 0.005$ & $0.117 \pm 0.002$ & -20.11 & - & 7.15 & - \\
\hline 29 & NGC 3377 & $0.62^{b}$ & $0.36^{a}$ & 3.37 & $139^{a}$ & $0.446 \pm 0.001$ & $0.343 \pm 0.006$ & -20.07 & 10.02 & 4.55 & 0.522 \\
\hline 30 & NGC 3384 & $0.71^{b}$ & $0.36^{a}$ & 4.22 & $148^{a}$ & $0.995 \pm 0.001$ & $0.251 \pm 0.001$ & -19.93 & 10.93 & 14.90 & 0.397 \\
\hline 31 & NGC 3412 & $0.67^{c}$ & $0.73^{c}$ & 3.40 & - & $0.146 \pm 0.035$ & $0.254 \pm 0.001$ & -19.96 & - & 11.29 & 0.403 \\
\hline 32 & NGC 3414 & $0.84^{b}$ & $0.81^{a}$ & 3.82 & $237^{a}$ & $1.746 \pm 0.002$ & $0.216 \pm 0.001$ & -20.25 & 10.94 & 6.07 & 0.070 \\
\hline
\end{tabular}




\begin{tabular}{|c|c|c|c|c|c|c|c|c|c|c|c|}
\hline 33 & NGC 3458 & $0.59^{c}$ & $1.17^{c}$ & 3.18 & - & $-0.146 \pm 0.037$ & $0.120 \pm 0.001$ & -19.86 & - & 8.41 & 0.250 \\
\hline 34 & NGC 3489 & $0.57^{c}$ & $0.72^{c}$ & 2.84 & - & $-0.238 \pm 0.043$ & $0.250 \pm 0.002$ & -19.30 & - & 4.64 & 0.552 \\
\hline 35 & NGC 3595 & $0.76^{b}$ & $0.93^{a}$ & 3.39 & - & $-0.316 \pm 0.004$ & $0.343 \pm 0.001$ & -20.96 & - & 2.87 & 0.301 \\
\hline 36 & NGC 3599 & $0.75^{b}$ & $0.65^{a}$ & 3.77 & $85^{a}$ & $0.264 \pm 0.003$ & $0.115 \pm 0.001$ & -19.93 & 10.00 & 7.45 & 0.239 \\
\hline 37 & NGC 3605 & $0.60^{b}$ & $0.65^{a}$ & 2.52 & $92^{a}$ & $-0.734 \pm 0.037$ & $0.261 \pm 0.001$ & -19.61 & 8.81 & 2.23 & 0.347 \\
\hline 38 & NGC 3610 & $0.76^{b}$ & $0.64^{a}$ & 3.28 & $162^{a}$ & $2.128 \pm 0.003$ & $0.437 \pm 0.002$ & -20.96 & 10.07 & 3.96 & 0.539 \\
\hline 39 & NGC 3796 & $0.74^{c}$ & $1.04^{c}$ & 2.88 & - & $0.125 \pm 0.052$ & $0.370 \pm 0.001$ & -18.66 & - & 10.39 & 0.119 \\
\hline 40 & NGC 3900 & $1.02^{b}$ & $1.16^{a}$ & 3.61 & $118^{a}$ & $0.294 \pm 0.003$ & $0.233 \pm 0.002$ & -20.80 & 10.12 & 2.25 & - \\
\hline 41 & NGC 3945 & $0.57^{b}$ & $0.59^{a}$ & 3.37 & $174^{a}$ & $2.645 \pm 0.003$ & $0.230 \pm 0.001$ & -20.25 & 10.22 & 5.03 & 0.561 \\
\hline 42 & NGC 4026 & $0.65^{b}$ & $0.48^{a}$ & 3.26 & $178^{a}$ & $4.249 \pm 0.003$ & $0.368 \pm 0.002$ & -19.79 & 10.13 & 2.52 & 0.442 \\
\hline 43 & NGC 4121 & $0.85^{b}$ & $0.79^{a}$ & 2.73 & $86^{a}$ & $-0.085 \pm 0.005$ & $0.242 \pm 0.001$ & -18.53 & 8.97 & 1.32 & - \\
\hline 44 & NGC 4128 & $0.75^{b}$ & $0.92^{a}$ & 3.48 & $203^{a}$ & $-0.498 \pm 0.042$ & $0.370 \pm 0.001$ & -20.79 & 10.46 & 9.25 & - \\
\hline 45 & NGC 4143 & $0.61^{b}$ & $0.88^{a}$ & 3.09 & $214^{a}$ & $0.714 \pm 0.002$ & $0.228 \pm 0.001$ & -19.68 & 10.12 & 2.62 & 0.398 \\
\hline 46 & NGC 4150 & $0.68^{b}$ & $0.85^{a}$ & 3.49 & $85^{a}$ & $0.084 \pm 0.002$ & $0.209 \pm 0.002$ & -18.66 & 9.72 & 10.40 & 0.338 \\
\hline 47 & NGC 4203 & $0.74^{c}$ & $0.85^{c}$ & 2.98 & - & $0.655 \pm 0.028$ & $0.082 \pm 0.001$ & -19.83 & - & 4.89 & 0.275 \\
\hline 48 & NGC 4262 & $0.76^{c}$ & $0.87^{c}$ & 2.81 & - & $0.228 \pm 0.025$ & $0.099 \pm 0.001$ & -20.04 & - & 4.31 & 0.250 \\
\hline 49 & NGC 4267 & $0.71^{c}$ & $0.88^{c}$ & 2.92 & - & $0.769 \pm 0.024$ & $0.073 \pm 0.001$ & -19.78 & - & 3.94 & 0.253 \\
\hline 50 & NGC 4281 & $0.56^{c}$ & $1.04^{c}$ & 4.20 & - & $0.774 \pm 0.041$ & $0.463 \pm 0.001$ & -21.84 & - & 12.00 & 0.621 \\
\hline 51 & NGC 4283 & $0.80^{c}$ & $1.04^{c}$ & 2.75 & - & $-0.091 \pm 0.031$ & $0.052 \pm 0.001$ & -18.72 & - & 3.39 & 0.151 \\
\hline 52 & NGC 4339 & $0.81^{c}$ & $0.87^{c}$ & 3.59 & - & $-0.020 \pm 0.033$ & $0.051 \pm 0.001$ & -19.95 & - & 7.30 & 0.312 \\
\hline 53 & NGC 4340 & $0.68^{c}$ & $0.89^{c}$ & 3.94 & - & $0.558 \pm 0.131$ & $0.192 \pm 0.003$ & -19.44 & - & 12.35 & 0.442 \\
\hline 54 & NGC 4342 & $0.55^{c}$ & $0.84^{c}$ & 3.23 & $219^{e}$ & $3.559 \pm 0.102$ & $0.445 \pm 0.002$ & -18.13 & 10.28 & 8.00 & 0.306 \\
\hline 55 & NGC 4387 & $0.65^{b}$ & $0.54^{a}$ & 2.71 & $104^{a}$ & $-1.414 \pm 0.003$ & $0.304 \pm 0.001$ & -19.25 & 9.11 & 2.35 & 0.317 \\
\hline 56 & NGC 4417 & $0.75^{b}$ & $0.94^{a}$ & 3.24 & $131^{a}$ & $1.868 \pm 0.002$ & $0.345 \pm 0.001$ & -18.94 & 9.84 & 4.68 & 0.392 \\
\hline 57 & NGC 4429 & $1.07^{c}$ & $0.58^{c}$ & 4.33 & - & $-0.1344 \pm 0.044$ & $0.442 \pm 0.001$ & -20.41 & - & 9.35 & 0.396 \\
\hline 58 & NGC 4434 & $0.64^{b}$ & $0.54^{a}$ & 3.00 & $120^{a}$ & $0.124 \pm 0.003$ & $0.062 \pm 0.001$ & -19.19 & 9.52 & 3.91 & 0.199 \\
\hline 59 & NGC 4442 & $0.52^{c}$ & $0.72^{c}$ & 2.97 & $59^{e}$ & $-0.622 \pm 0.032$ & $0.285 \pm 0.001$ & -19.04 & 8.88 & 4.90 & 0.338 \\
\hline 60 & NGC 4464 & $0.70^{b}$ & $0.54^{a}$ & 2.80 & $127^{a}$ & $0.714 \pm 0.003$ & $0.280 \pm 0.001$ & -18.82 & 9.37 & 3.24 & - \\
\hline 61 & NGC 4467 & $0.94^{b}$ & $0.54^{a}$ & 2.91 & $68^{a}$ & $0.606 \pm 0.007$ & $0.285 \pm 0.001$ & -17.51 & 8.94 & 4.00 & - \\
\hline 62 & NGC 4474 & $0.72^{b}$ & $0.72^{a}$ & 3.45 & $87^{a}$ & $2.271 \pm 0.004$ & $0.267 \pm 0.001$ & -18.42 & 9.70 & 3.93 & 0.353 \\
\hline 63 & NGC 4483 & $0.88^{c}$ & $0.91^{c}$ & 3.38 & $92^{e}$ & $-0.245 \pm 0.056$ & $0.262 \pm 0.001$ & -18.44 & 9.68 & 8.27 & 0.273 \\
\hline 64 & NGC 4486A & $0.72^{c}$ & $0.95^{c}$ & 2.98 & - & $-0.046 \pm 0.072$ & $0.244 \pm 0.005$ & -18.92 & - & 5.16 & 0.351 \\
\hline 65 & NGC 4489 & $0.64^{c}$ & $0.87^{c}$ & 4.41 & $62^{e}$ & $0.174 \pm 0.061$ & $0.088 \pm 0.001$ & -18.64 & 10.37 & 9.39 & 0.117 \\
\hline 66 & NGC 4494 & $0.55^{b}$ & $0.54^{a}$ & 3.68 & $150^{a}$ & $0.032 \pm 0.001$ & $0.148 \pm 0.001$ & -21.50 & 10.40 & 3.69 & 0.212 \\
\hline 67 & NGC 4503 & $0.65^{b}$ & $0.63^{a}$ & 4.28 & $111^{a}$ & $0.029 \pm 0.002$ & $0.270 \pm 0.001$ & -19.57 & 10.74 & 7.69 & 0.470 \\
\hline 68 & NGC 4528 & $0.97^{c}$ & $0.88^{c}$ & 2.94 & - & $-0.615 \pm 0.059$ & $0.169 \pm 0.002$ & -19.72 & - & 3.72 & 0.102 \\
\hline 69 & NGC 4550 & $0.57^{c}$ & $0.88^{c}$ & 2.59 & - & $2.086 \pm 0.086$ & $0.582 \pm 0.001$ & -17.32 & - & 1.94 & 0.061 \\
\hline 70 & NGC 4551 & $0.69^{b}$ & $0.54^{a}$ & 3.12 & $108^{a}$ & $-0.456 \pm 0.002$ & $0.260 \pm 0.001$ & -19.37 & 9.55 & 2.14 & 0.259 \\
\hline 71 & NGC 4564 & $0.81^{b}$ & $0.63^{a}$ & 3.36 & $157^{a}$ & $1.426 \pm 0.002$ & $0.333 \pm 0.001$ & -20.26 & 10.12 & 4.50 & 0.536 \\
\hline 72 & NGC 4570 & $0.85^{c}$ & $0.92^{c}$ & 3.51 & - & $1.154 \pm 0.073$ & $0.396 \pm 0.001$ & -21.26 & - & 6.48 & 0.498 \\
\hline 73 & NGC 4578 & $0.89^{c}$ & $0.90^{c}$ & 3.81 & - & $0.342 \pm 0.033$ & $0.233 \pm 0.001$ & -21.01 & - & 7.66 & 0.544 \\
\hline 74 & NGC 4596 & $0.77^{c}$ & $0.90^{c}$ & 4.04 & - & $1.289 \pm 0.058$ & $0.216 \pm 0.001$ & -21.42 & - & 7.31 & 0.280 \\
\hline 75 & NGC 4612 & $0.64^{c}$ & $0.91^{c}$ & 4.60 & - & $-0.207 \pm 0.041$ & $0.192 \pm 0.001$ & -20.79 & - & 16.74 & 0.324 \\
\hline 76 & NGC 4621 & $0.85^{b}$ & $0.54^{a}$ & 3.43 & $225^{a}$ & $1.539 \pm 0.001$ & $0.325 \pm 0.001$ & -21.74 & 10.50 & 6.06 & 0.291 \\
\hline 77 & NGC 4623 & $2.06^{c}$ & $0.93^{c}$ & 3.59 & - & $1.112 \pm 0.100$ & $0.562 \pm 0.001$ & -19.80 & - & 5.02 & 0.564 \\
\hline 78 & NGC 4638 & $0.77^{c}$ & $0.93^{c}$ & 3.04 & - & $3.801 \pm 0.172$ & $0.538 \pm 0.002$ & -20.03 & - & 3.41 & 0.715 \\
\hline 79 & NGC 4660 & $0.91^{b}$ & $0.54^{a}$ & 2.99 & $188^{a}$ & $1.100 \pm 0.002$ & $0.345 \pm 0.001$ & -20.13 & 9.90 & 3.90 & 0.475 \\
\hline 80 & NGC 4754 & $0.60^{c}$ & $0.04^{c}$ & 3.70 & - & $-0.038 \pm 0.032$ & $0.182 \pm 0.001$ & -20.84 & - & 8.79 & 0.418 \\
\hline 81 & NGC 5173 & $0.52^{c}$ & $1.27^{c}$ & 3.48 & $99^{e}$ & $0.140 \pm 0.036$ & $0.121 \pm 0.001$ & -20.09 & 9.84 & 9.26 & 0.106 \\
\hline 82 & NGC 5273 & $1.66^{c}$ & $0.89^{c}$ & 3.85 & $90^{e}$ & $0.130 \pm 0.052$ & $0.135 \pm 0.001$ & -19.45 & 10.13 & 10.00 & 0.482 \\
\hline 83 & NGC 5308 & $0.96^{b}$ & $0.90^{a}$ & 3.51 & $211^{a}$ & $3.143 \pm 0.004$ & $0.467 \pm 0.001$ & -21.26 & 10.53 & 3.05 & 0.510 \\
\hline 84 & NGC 5370 & $0.67^{b}$ & $1.04^{a}$ & 3.54 & $133^{a}$ & $3.403 \pm 0.010$ & $0.294 \pm 0.002$ & -20.60 & 10.15 & 4.38 & - \\
\hline 85 & NGC 5831 & $0.55^{b}$ & $0.85^{a}$ & 3.67 & $164^{a}$ & $0.305 \pm 0.002$ & $0.272 \pm 0.001$ & -21.00 & 10.47 & 5.11 & 0.065 \\
\hline 86 & NGC 5838 & $0.93^{b}$ & $1.03^{a}$ & 3.52 & $266^{a}$ & $-0.057 \pm 0.002$ & $0.167 \pm 0.002$ & -20.51 & 10.74 & 4.73 & 0.460 \\
\hline 87 & NGC 5845 & $0.52^{b}$ & $1.14^{a}$ & 2.64 & $234^{a}$ & $-0.497 \pm 0.002$ & $0.255 \pm 0.001$ & -19.98 & 9.74 & 2.95 & 0.358 \\
\hline 88 & NGC 5854 & $1.01^{c}$ & $0.39^{c}$ & 3.76 & - & $-0.471 \pm 0.091$ & $0.370 \pm 0.001$ & -20.41 & - & 9.08 & 0.515 \\
\hline 89 & NGC 6278 & $0.67^{b}$ & $0.99^{a}$ & 3.66 & $150^{a}$ & $0.653 \pm 0.003$ & $0.233 \pm 0.001$ & -20.81 & 10.38 & 6.26 & 0.411 \\
\hline 90 & NGC 6340 & $0.64^{b}$ & $0.91^{a}$ & 3.56 & $144^{a}$ & $-0.174 \pm 0.002$ & $0.036 \pm 0.001$ & -19.46 & 10.24 & 15.97 & - \\
\hline 91 & NGC 7280 & $0.87^{c}$ & $1.06^{c}$ & 4.17 & - & $-0.485 \pm 0.062$ & $0.329 \pm 0.001$ & -20.37 & - & 9.98 & 0.503 \\
\hline
\end{tabular}


He et al.

\begin{tabular}{cccccccccccc}
92 & NGC 7332 & $0.80^{b}$ & $0.67^{a}$ & 3.20 & $124^{a}$ & $2.196 \pm 0.178$ & $0.487 \pm 0.002$ & -19.62 & 9.75 & 8.70 & 0.338 \\
93 & NGC 7743 & $0.57^{b}$ & $1.03^{a}$ & 3.60 & $84^{a}$ & $1.058 \pm 0.103$ & $0.293 \pm 0.001$ & -20.18 & 9.82 & 16.97 & - \\
94 & UGC 4551 & $0.51^{b}$ & $0.82^{a}$ & 2.89 & $167^{a}$ & $-0.156 \pm 0.002$ & $0.145 \pm 0.001$ & -19.78 & 9.70 & 3.10 & - \\
95 & UGC 4587 & $0.81^{b}$ & $1.05^{a}$ & 3.81 & - & $-0.378 \pm 0.006$ & $0.320 \pm 0.001$ & -20.77 & - & 4.77 & - \\
96 & UGC 6062 & $0.82^{b}$ & $1.01^{a}$ & 3.26 & $142^{e}$ & $0.339 \pm 0.005$ & $0.250 \pm 0.001$ & -20.34 & 9.93 & 3.53 & - \\
97 & VCC 1199 & $0.90^{b}$ & $0.54^{a}$ & 3.97 & $55^{e}$ & $0.484 \pm 0.015$ & $0.025 \pm 0.001$ & -15.58 & 9.82 & 10.00 & - \\
98 & VCC 1440 & $0.89^{b}$ & $0.54^{a}$ & 2.41 & - & $0.140 \pm 0.008$ & $0.129 \pm 0.002$ & -17.24 & - & 3.30 & - \\
99 & VCC 1545 & $0.51^{b}$ & $0.54^{a}$ & 3.30 & $51^{e}$ & $-0.215 \pm 0.012$ & $0.144 \pm 0.004$ & -17.49 & 9.08 & 2.89 & - \\
100 & VCC 1627 & $0.69^{b}$ & $0.54^{a}$ & 1.93 & - & $0.097 \pm 0.009$ & $0.089 \pm 0.002$ & -16.42 & - & 1.78 & - \\
\hline
\end{tabular}

Notes: See the notes in Table 1 for each column.

Table 3: "Intermediate" Galaxy Parameters

\begin{tabular}{|c|c|c|c|c|c|c|c|c|c|c|c|}
\hline (1) & Galaxy & $\begin{array}{l}\gamma^{\prime} \\
(3)\end{array}$ & $\begin{array}{c}r_{\gamma} \\
\log (\mathrm{pc}) \\
(4)\end{array}$ & $\begin{array}{c}R_{e} \\
\log (\mathrm{pc}) \\
(5)\end{array}$ & $\begin{array}{c}\sigma \\
\mathrm{km} \mathrm{s}^{-1} \\
(6)\end{array}$ & $\begin{array}{c}a_{4} / a \\
10^{(-2)} \\
(7) \\
\end{array}$ & (8) & $\begin{array}{l}M_{V} \\
\operatorname{mag} \\
(9)\end{array}$ & $\begin{array}{c}\log \frac{M_{d y n}}{M_{\odot}} \\
(10)\end{array}$ & $(11)$ & $\lambda_{R_{e} / 2}$ \\
\hline 1 & J091944.2+562201.1 & $0.32^{d}$ & - & 4.97 & $327^{e}$ & $-1.982 \pm 0.134$ & $0.198 \pm 0.020$ & -23.84 & 12.37 & 6.46 & - \\
\hline 2 & $\mathrm{~J} 112842.0+043221.7$ & $0.47^{d}$ & - & 4.01 & $360^{e}$ & $-1.068 \pm 0.194$ & $0.249 \pm 0.021$ & -22.86 & 11.49 & 3.09 & - \\
\hline 3 & $\mathrm{~J} 120011.1+680924.8$ & $0.33^{d}$ & - & 4.69 & $380^{e}$ & $1.249 \pm 0.078$ & $0.310 \pm 0.009$ & -23.95 & 12.22 & 4.66 & - \\
\hline 4 & $\mathrm{~J} 133724.7+033656.5$ & $0.37^{d}$ & - & 4.83 & $414^{e}$ & $-1.755 \pm 0.079$ & $0.167 \pm 0.008$ & -22.50 & 12.43 & 10.05 & - \\
\hline 5 & $\mathrm{~J} 135602.4+021044.6$ & $0.40^{d}$ & - & 4.26 & $352^{e}$ & $-1.807 \pm 0.109$ & $0.272 \pm 0.010$ & -23.89 & 11.72 & 4.89 & - \\
\hline 6 & $\mathrm{~J} 162332.4+450032.0$ & $0.35^{d}$ & - & 4.65 & $356^{e}$ & $0.142 \pm 0.068$ & $0.202 \pm 0.007$ & -23.14 & 12.12 & 4.82 & - \\
\hline 7 & NGC 2841 & $0.34^{b}$ & $1.09^{a}$ & 3.76 & $206^{a}$ & $1.778 \pm 0.001$ & $0.286 \pm 0.001$ & -20.57 & 10.75 & 12.98 & - \\
\hline 8 & NGC 3998 & $0.49^{c}$ & $0.83^{c}$ & 2.71 & - & $0.424 \pm 0.066$ & $0.147 \pm 0.002$ & -19.58 & - & 2.17 & 0.342 \\
\hline 9 & NGC 4239 & $0.46^{b}$ & $1.06^{a}$ & 3.03 & $62^{a}$ & $1.051 \pm 0.004$ & $0.420 \pm 0.001$ & -18.50 & 8.98 & 2.56 & - \\
\hline 10 & NGC 4270 & $0.44^{c}$ & $1.62^{c}$ & 3.66 & - & $-0.527 \pm 0.048$ & $0.420 \pm 0.001$ & -20.79 & - & 5.75 & 0.294 \\
\hline 11 & NGC 4350 & $0.47^{c}$ & $1.57^{c}$ & 3.24 & - & $2.411 \pm 0.132$ & $0.415 \pm 0.001$ & -20.44 & - & 5.00 & $\begin{array}{l}0.294 \\
0.480\end{array}$ \\
\hline 12 & NGC 4377 & $0.41^{c}$ & $1.17^{c}$ & 3.75 & - & $0.587 \pm 0.035$ & $0.157 \pm 0.001$ & -19.90 & - & 15.19 & 0.338 \\
\hline 13 & NGC 4379 & $0.46^{c}$ & $1.02^{c}$ & 3.18 & _- & $0.442 \pm 0.031$ & $0.182 \pm 0.001$ & -19.47 & _ & 5.04 & 0.300 \\
\hline 14 & NGC 4452 & $0.39^{c}$ & $2.37^{c}$ & 2.38 & $45^{e}$ & $2.301 \pm 0.287$ & $0.667 \pm 0.008$ & -15.52 & 8.05 & 1.22 & 0.648 \\
\hline 15 & NGC 4476 & $0.34^{c}$ & $2.32^{c}$ & 3.25 & $49^{e}$ & $2.432 \pm 0.175$ & $0.426 \pm 0.003$ & -20.27 & 9.01 & 2.92 & 0.266 \\
\hline 16 & NGC 4477 & $0.38^{c}$ & $1.38^{c}$ & 3.43 & - & $2.203 \pm 0.069$ & $0.218 \pm 0.001$ & -21.05 & - & 4.02 & 0.221 \\
\hline 17 & NGC 4482 & $0.49^{b}$ & $2.05^{a}$ & 3.59 & $26^{a}$ & $-0.260 \pm 0.005$ & $0.324 \pm 0.001$ & -18.87 & 8.79 & 2.40 & - \\
\hline 18 & NGC 4733 & $0.35^{c}$ & $1.90^{c}$ & 3.55 & $\begin{array}{r}20 \\
-\end{array}$ & $1.240 \pm 0.066$ & $0.224 \pm 0.001$ & $\begin{array}{l}-10.01 \\
-18.70\end{array}$ & $\begin{array}{c}0.19 \\
-\end{array}$ & $\begin{array}{l}2.40 \\
4.60\end{array}$ & $0 . \overline{076}$ \\
\hline 19 & NGC 4762 & $0.40^{c}$ & $1.42^{c}$ & 3.13 & - & $2.335 \pm 0.056$ & $0.513 \pm 0.002$ & -20.20 & - & 7.10 & 0.724 \\
\hline 20 & NGC 5422 & $0.45^{c}$ & $1.30^{c}$ & 3.97 & - & $2.186 \pm 0.076$ & $0.385 \pm 0.002$ & -20.19 & - & 12.22 & 0.501 \\
\hline 21 & NGC 5475 & $0.40^{c}$ & $1.49^{c}$ & 3.76 & $102^{e}$ & $0.874 \pm 0.090$ & $0.450 \pm 0.001$ & -19.78 & 10.15 & 9.88 & 0.638 \\
\hline
\end{tabular}

Notes: See the notes in Table 1 for each column.

\section{RESULTS AND DISCUSSIONS}

\subsection{The relations of $a_{4} / a$ with central properties of ETGs}

In this subsection, we re-visit the relations between the isophotal shape described by characteristic parameter $a_{4} / a$ and the central photometric properties of ETGs. Figure1 1 shows the distributions of $a_{4} / a$ for "core" and "power-law" ETGs. It is clear from the histograms shown in Figure 1 that the distribution of $a_{4} / a$ for "core" and "power-law" ETGs is not significantly separated, i.e. the $a_{4} / a$ distribution of a large fraction of "core" and "power-law" ETGs overlap. The fractions of boxy and disky galaxies are $59 \%(37 / 63)$ and $41 \%(26 / 63)$ for "core" ETGs, and the median value of $a_{4} / a$ is $-0.26 \times 10^{-3}$. While for the 100 "power-law" ETGs, the fractions of boxy and disky galaxies are 35\% (35/100) and 65\% (65/100), respectively, and $a_{4} / a$ has the median value of $1.88 \times 10^{-3}$. It suggests that "core" galaxies are not necessarily boxy, and only two-thirds "power-law" galaxies have disky deviated isophotes. But the most deviated disky galaxies are "power-law" galaxies.

The left panel of Figure2 2 shows the $a_{4} / a$ as a function of $\gamma^{\prime}$ that describes the slope of the central surface brightness profile of ETGs (see Lauer et al. 2007b). The two dotted vertical lines in Figure 2 


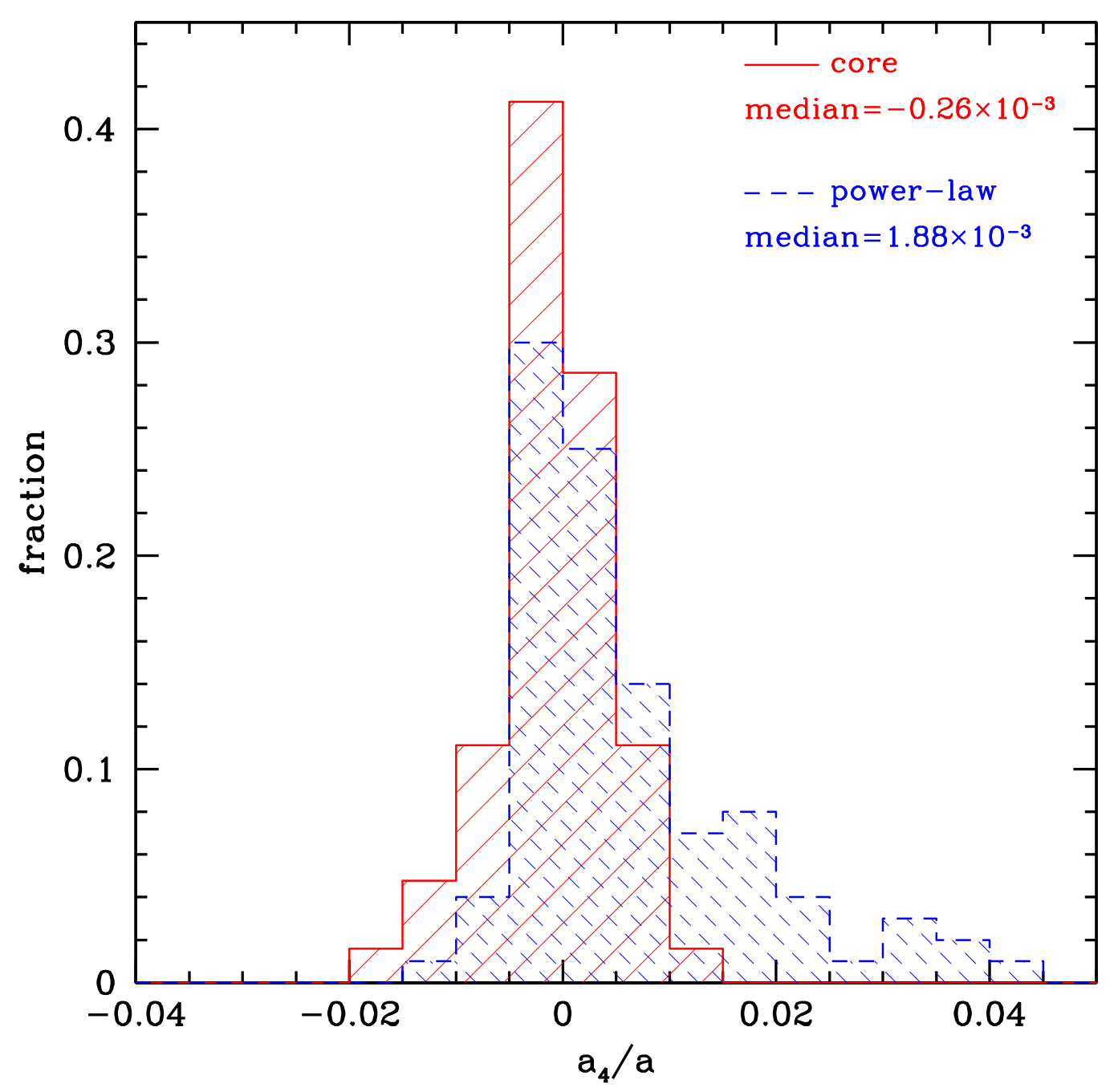

Fig. 1 Histograms of the isophotal shape characteristic parameter $a_{4} / a$ for "core" (red solid line) and "power-law" (blue dashed line) ETGs. The median values are indicated in the top right.

divide sample ETGs into "core", "intermediate" and "power-law" galaxies, while the disky and boxy ETGs are located above and below the horizontal dotted line. Although, there is a trend that the values of $a_{4} / a$ increase as $\gamma^{\prime}$ increasing, the scatter shown in Figure 2 is quite large, i.e. the correlation between $a_{4} / a$ and $\gamma^{\prime}$ is weak. The Spearman rank-order correlation coefficient between $a_{4} / a$ and $\gamma^{\prime}$ is $r_{s}=0.24$ and the probability that no correlation exists between these two parameters is $1.12 \times 10^{-3}$. Apart from the central light profiles slope $\gamma^{\prime}$, the values of $r_{\gamma}$ characterize the physical scale of the core of ETGs ("cusp radius", Lauer et al. 2007a). Lauer et al. (2007a) claimed that the core size $r_{\gamma}$ is tightly correlated with the galaxy luminosity $L$ and the black hole mass $M_{\bullet}$. However, from the right panel of Figure 2 


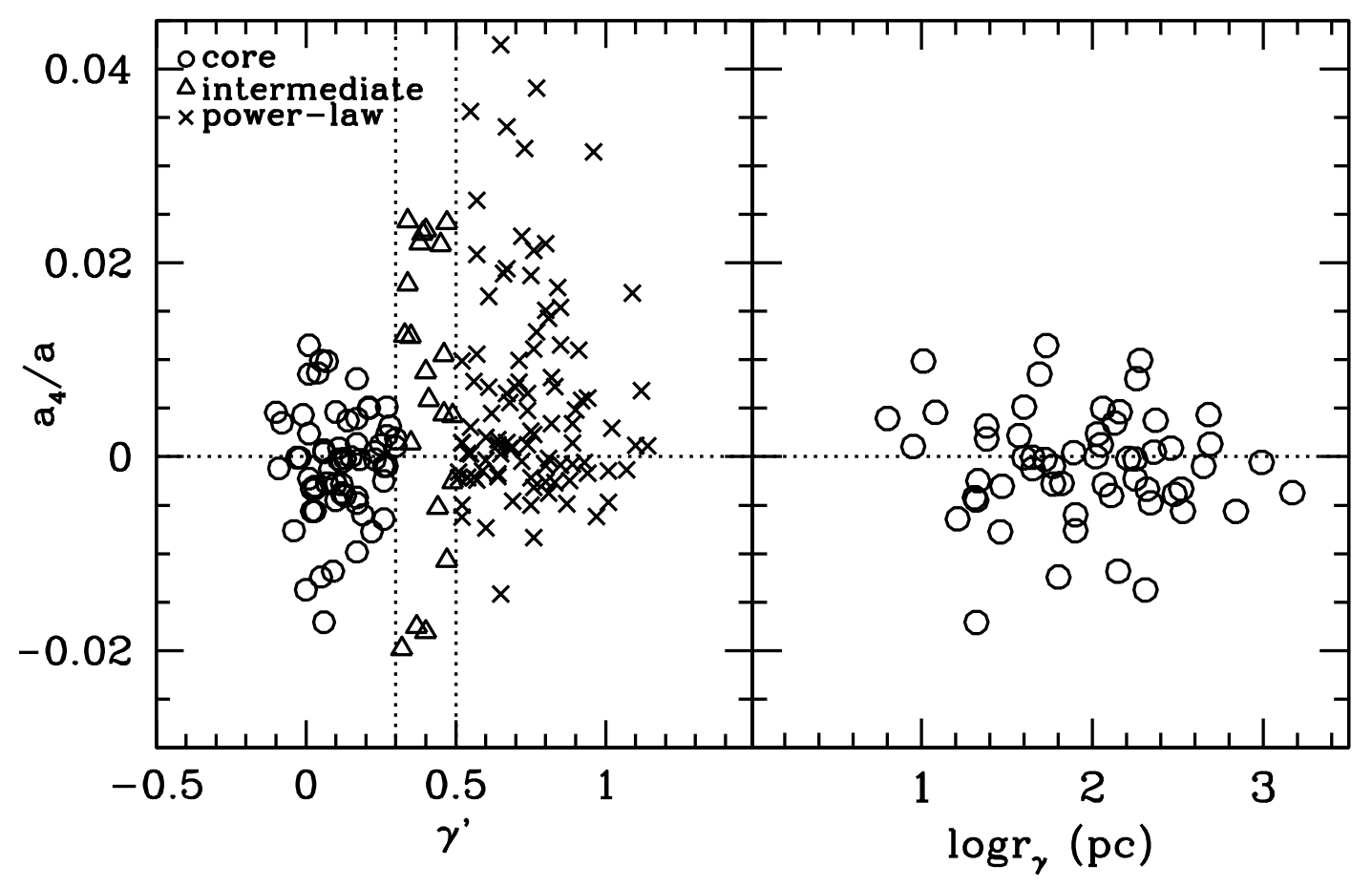

Fig. 2 Isophotal shape characteristic parameter $a_{4} / a$ as a function of (a) central surface brightness profile slope $\gamma^{\prime}$ (left panel) and (b) "cusp radius" $r_{\gamma}$ for "core" galaxies (right panel). The horizontal dotted lines show the classification of disky $\left(a_{4} / a>0\right)$ and boxy $\left(a_{4} / a<0\right)$ isophotal shape, while the vertical dotted lines in the left panel show the separation of "core" $\left(\gamma^{\prime}<0.3\right.$, circles $)$, "power-law" $\left(\gamma^{\prime}>0.5\right.$, crosses $)$ and "intermediate" ETGs $\left(0.3<\gamma^{\prime}<0.5\right.$, triangles $)$.

that shows the $a_{4} / a$ as a function of $r_{\gamma}$, there is no correlation found between $a_{4} / a$ and $r_{\gamma}$ for "core" ETGs.

Note that Bender et al. (1989) pointed out that $a_{4} / a$ might be influenced by the projection effect, but such effect could only lead to the change on its absolute value, but not the sign of $a_{4} / a$. Thus the classification of boxy and disky ETGs reflects the intrinsic isophotal property of ETGs. Therefore 
the statistical results shown above do not support the statement that there is a close relation between isophotal shape and their central light profile for ETGs.

\subsection{The relations of $a_{4} / a$ with global properties of ETGs}

In this subsection, we investigate the relations of the isophotal shape with other global physical properties for "core" and "power-law" ETGs. The left panel of Figure 3 shows $a_{4} / a$ as a function of the $V$-band luminosity $\left(M_{V}\right)$. It can be seen from the left panel of Figure 3 that there is a weak correlation between $a_{4} / a$ and $M_{V}$ for the whole sample ETGs, indicating that boxy (disky) ETGs tend to be bright (faint). Faber et al. (1997) pointed out that luminous galaxies with $M_{V}<-22$ mag have shallow "core" inner profiles, faint galaxies with $M_{V}>-20.5$ mag show steep "power-law" inner profiles, and for those with $-22<M_{V}<-20.5$, "core" and "power-law" galaxies coexist. Therefore, it is interesting to visit the fractions of boxy and disky galaxies in these luminosity intervals. The fraction of boxy (disky) galaxies is $\sim 32 \%$ (68\%) for faint ETGs with $M_{V}>-20.5 \mathrm{mag}$, while $\sim 58 \%$ (42\%) for the most luminous ETGs with $M_{V}<-22$ mag. For ETGs in the luminosity interval of $-22<M_{V}<-20.5$, the fractions of boxy and disky galaxies are similar ( $\sim 46 \%$ and 54\%, respectively). These results are consistent with Hao et al. (2006). On the other hand, the fraction of "core" ("power-law") is 7\% (79\%) for faint ETGs with $M_{V}>-20.5 \mathrm{mag}$, while $\sim 74 \%$ (14\%) for the most luminous ETGs with $M_{V}<-22 \mathrm{mag}$. In the luminosity interval of $-22<M_{V}<-20.5$, the fractions are comparable for "core" and "power-law" ETGs ( $\sim 41 \%$ and 53\%, respectively). Thus we confirm the conclusion of Faber et al. (1997). In the right panel of Figure 3, we show the isophotal shape parameter $a_{4} / a$ as a function of the dynamical mass. Similar to the left panel of Figure 3, there is tendency that $a_{4} / a$ decreases as ETGs are more massive and "core" ETGs are dominated by massive ETGs with dynamical mass larger than $10^{11} M_{\odot}$. Therefore, the bimodal classification based on the central light profile ("core" and "power-law") is more tightly correlated with galaxy luminosity and dynamical mass than that based on isophotal shape (boxy and disky).

As is well known, the Sérsic law is widely used to model the surface brightness profiles of ETGs and the best fitting value of Sérsic index $n$ could be used to describe the structures of galaxies. Based on photometric analysis for an ETGs sample in the Virgo cluster, Kormendy (2009a) claimed that giant ETGs characterized by $n>4$ tend to be rotating slowly, less flattened (ellipticity $\sim 0.15$ ) and with boxy isophotes as well as with "core" in their center. To test whether this argument applies to our ETGs sample, we plot $a_{4} / a$ as a function of the Sérsic index $n$ in Figure 4 It shows that there is no correlation between $a_{4} / a$ and the Sérsic index $n$ and the Sérsic index $n$ is smaller than 4 for quite a few of ETGs with "core" or boxy isophotes, which indicates that the central and isophotal properties of ETGs are not directly related to the Sérsic index $n$.

Moreover, ATLAS ${ }^{3 D}$ group (e.g. Cappellari et al. 2011) claimed that both isophotal shape and central light profile of ETGs are secondary indicators of the galaxy kinematic structure. They strongly suggest using the specific angular momentum parameter $\lambda_{R}$ as a discriminator of the bimodal distribution of ETGs, i.e. fast and slow rotators. It is interesting to investigate relations among isophotal shapes, central light profiles and the kinematic properties as parametrized by $\lambda_{R_{e} / 2}$ that is the $\lambda_{R}$ measured within half of the effective radius $R_{e}$. For our sample ETGs, the specific angular momentum $\lambda_{R_{e} / 2}$ is available for 111 objects from ATLAS ${ }^{3 \mathrm{D}}$. The sample size is 1.8 times that of Lauer (2012) who only compared the central light profile to $\lambda_{R_{e} / 2}$. Figure 5 shows how $a_{4} / a$ varies with $\lambda_{R_{e} / 2}$ for "core" and "power-law" ETGs. The dotted vertical line represents the $\lambda_{R_{e} / 2}=0.25$ line dividing slow and fast rotators as suggested by Lauer (2012), while the horizontal dotted line divides boxy and disky galaxies. It can be clearly seen that there is a trend that $a_{4} / a$ increases as $\lambda_{R_{e} / 2}$ increasing and the ETGs with highest $a_{4} / a$ are fast rotators. However, we can also see that such trend is only for power-law ETGs. We note that $a_{4} / a$ and $\lambda_{R_{e} / 2}$ are both affected by inclination effects. But inclination does not change the classification by isophotal shape or $\lambda_{R_{e} / 2}$ (Bender et al. 1989; Krajnović et al. 2013). So number statistics are more meaningful than the trend. The fraction of disky (boxy) galaxies is $\sim 70 \%(30 \%)$ for fast rotators $\left(\lambda_{R_{e} / 2}>0.25\right)$, while $\sim 44 \%(56 \%)$ for slow rotators $\left(\lambda_{R_{e} / 2}<0.25\right)$. Virtually Emsellem et al. (2011) already investigated the relation between isophotal shape parameter $a_{4} / a$ and kinematics of 


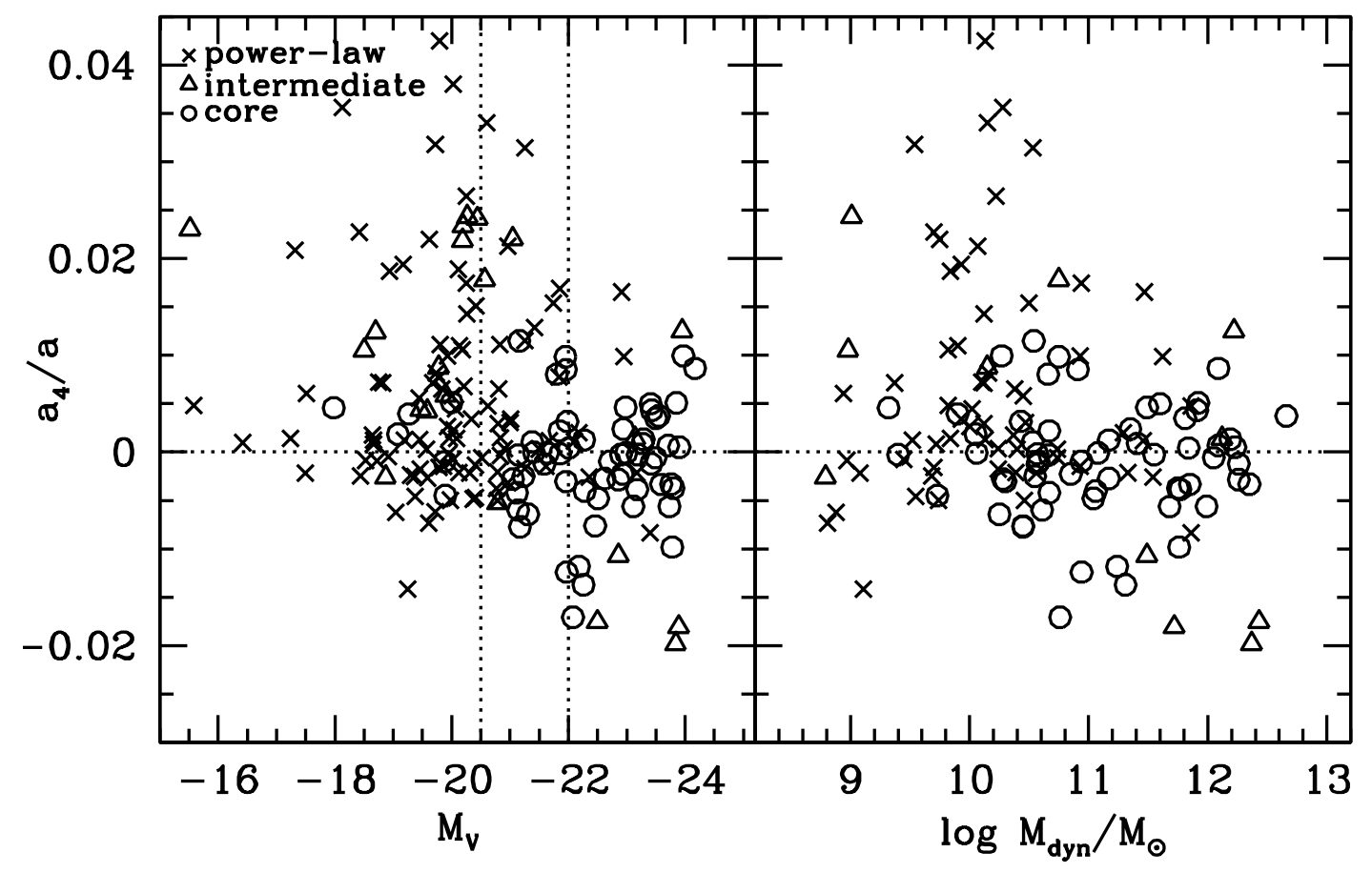

Fig. 3 Isophotal shape parameter $a_{4} / a$ versus the $V$-band absolute magnitude $M_{V}$ (left panel) and the dynamical mass (right panel). Circles represent "core" galaxies, triangles are "intermediate" galaxies, and crosses show "power-law" galaxies. The horizontal dotted line is the separation of disky $\left(a_{4} / a>0\right)$ and boxy $\left(a_{4} / a<0\right)$ ETGs. The vertical dotted lines in the left panel indicate the less luminous galaxies with $M_{V}>-20.5$, luminous galaxies with $M_{V}<-22$ and ETGs in the interval of $-22<M_{V}<-20.5$ reported by Lauer et al. (2007b).

ETGs as characterized by $\lambda_{R e}^{N}=\lambda_{R_{e}} / \sqrt{\epsilon}$, and concluded that there is not a simple correlation between these two parameters. Note that $\lambda_{R e}^{N}$ has been corrected for the inclination effects and $\lambda_{R e}^{N}=0.31$ was used as a separator of slow and fast rotators by Emsellem et al. (2011). We also examined the relation between $a_{4} / a$ and $\lambda_{R e}^{N}$ based on our sample ETGs, and found a similar result to Emsellem et al. (2011). But interestingly the fractions of disky/boxy galaxies in fast/slow rotators, as classified by 


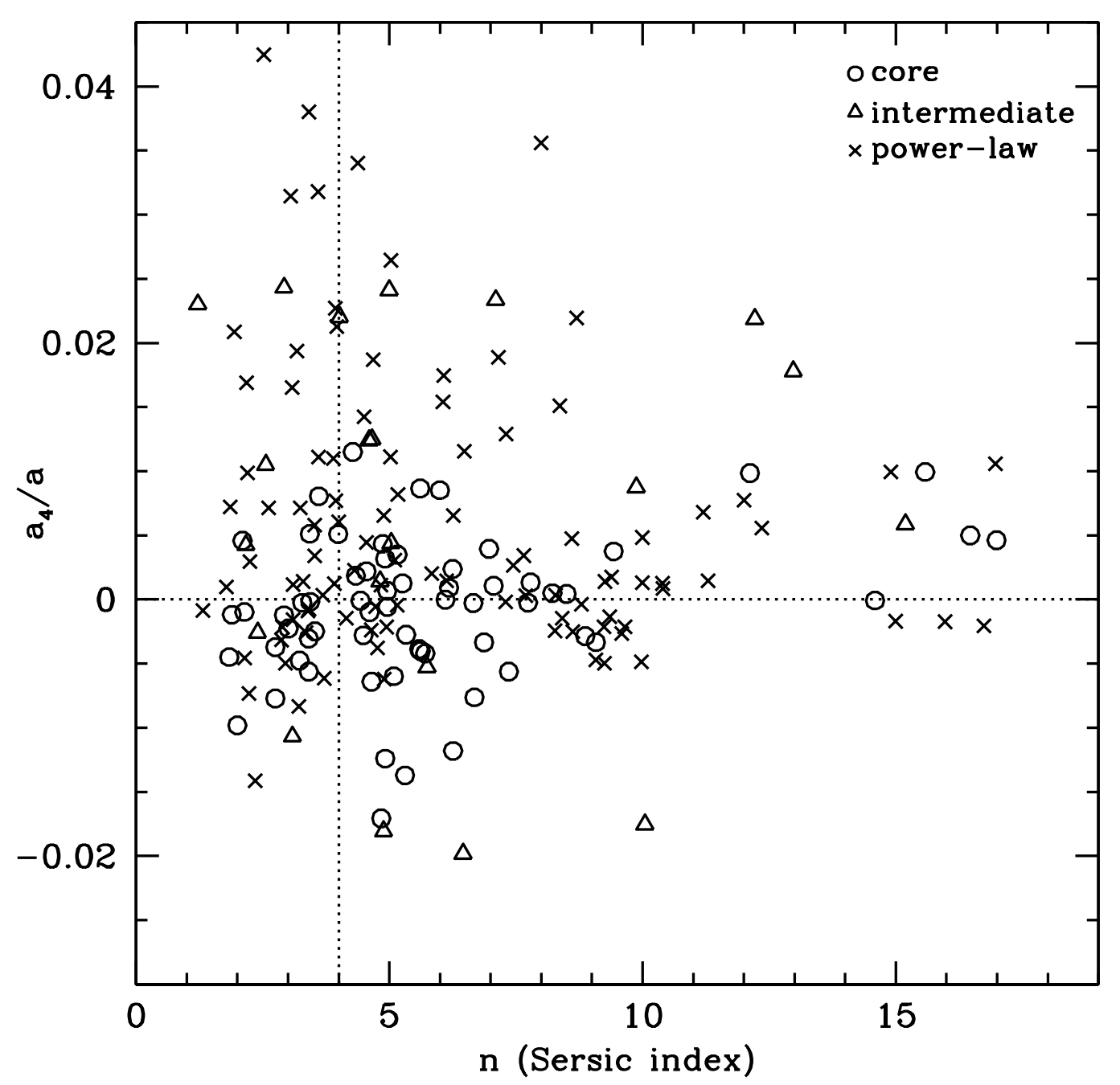

Fig. 4 Isophotal shape parameter $a_{4} / a$ versus Sérsic index n. Circles represent "core" galaxies, triangles are "intermediate" galaxies, and crosses show "power-law" galaxies. The horizontal dotted line is the separation of disky $\left(a_{4} / a>0\right)$ and boxy $\left(a_{4} / a<0\right)$ galaxies. The vertical dotted line indicates ETGs with $n=4$ following Kormendy (2009a).

$\lambda_{R e}^{N}=0.31$, are similar to those classified by $\lambda_{R_{e} / 2}=0.25$. Therefore, possible physical connection exists between $a_{4} / a$ and kinematic properties of ETGs whether the characteristic angular momentum parameter is influenced by projection effect.

The ATLAS ${ }^{3 D}$ group (e.g. Krajnović et al. 2013) and Lauer (2012) both found that $\lambda_{R}$ is correlated with the ellipticity $\epsilon$, and the distribution of "core" and "power-law"galaxies can be well separated in the $\lambda-\epsilon$ diagram. It is interesting to study the relations among the isophotal shapes, ellipticity and nuclear profiles of galaxies. Figure 6 shows $a_{4} / a$ as a function of $\epsilon$ for "core" and "power-law" ETGs. It shows that there is a weak correlation between $a_{4} / a$ and the ellipticity $\epsilon$ for "power-law" ETGs, 


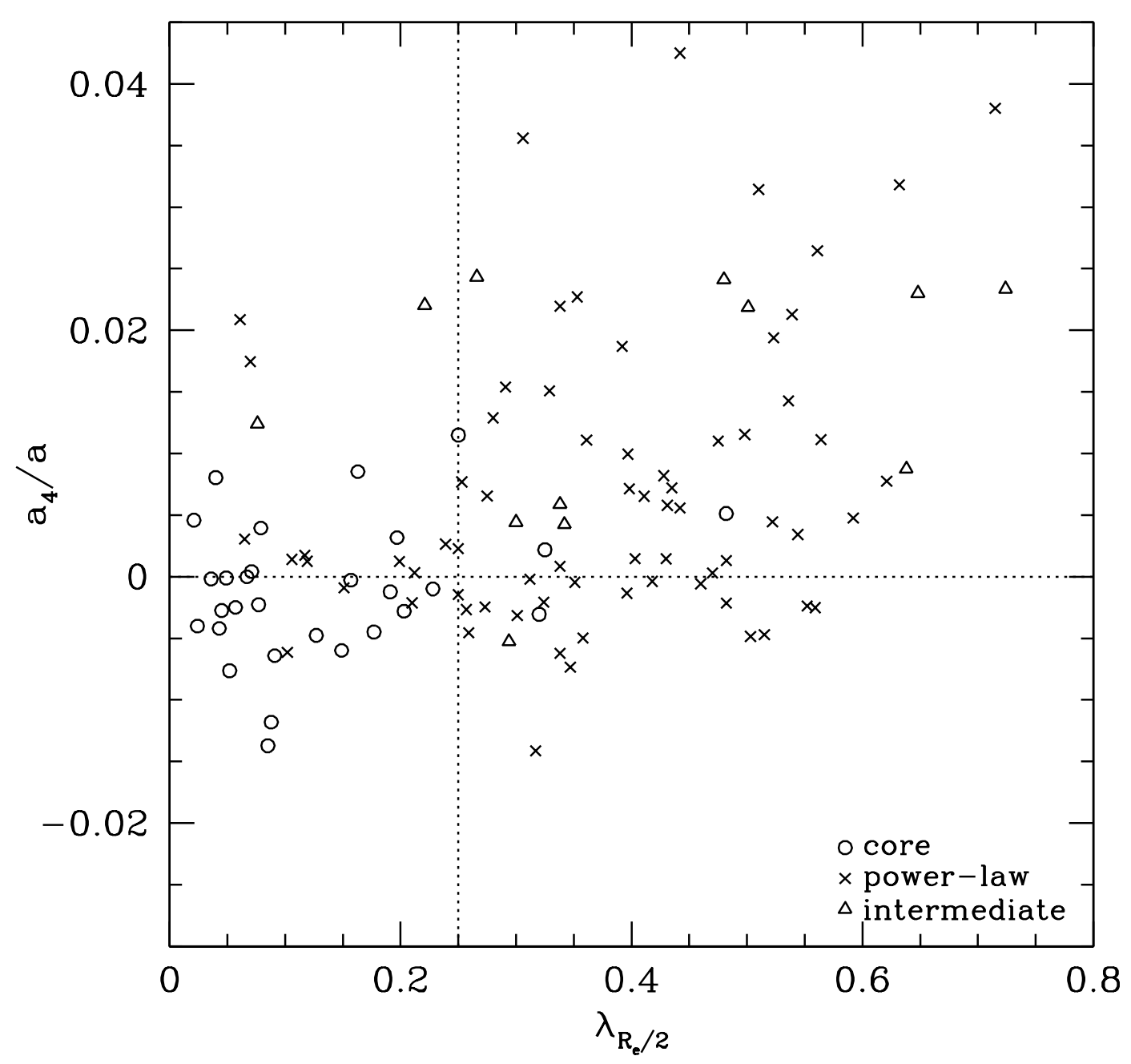

Fig. 5 Isophotal shape parameter $a_{4} / a$ as a function of specific angular momentum $\lambda_{R_{e} / 2}$. The circles show "core" galaxies, triangles are "intermediate" galaxies, and crosses show "power-law" galaxies. The horizontal dotted line separates disky $\left(a_{4} / a>0\right)$ and boxy $\left(a_{4} / a<0\right)$ galaxies. The vertical dotted line shows the discriminator of $\lambda_{R_{e} / 2}=0.25$ between slow and fast rotators.

and no correlation between these two parameters for "core" ETGs. From Figure 6, we note that the most distorted disky galaxies have the largest ellipticity, which is coincident with that found by Hao et al. (2006). In that work, this was explained as a consequence of a biased viewing angle. However, as shown in Figure 1 and Figure 5, the ETGs with largest $a_{4} / a$ are also "power-law" and fast rotators. So orientation cannot account for the high value of $a_{4} / a$, which may be caused by some physical processes. Specially, for those 111 ETGs that have $\lambda_{R_{e} / 2}$ measurements available from ATLAS ${ }^{3 \mathrm{D}}$, the Spearman rank-order correlation coefficients and the probabilities that no correlation exists for $\lambda_{R_{e} / 2}$ versus $\epsilon$ and 


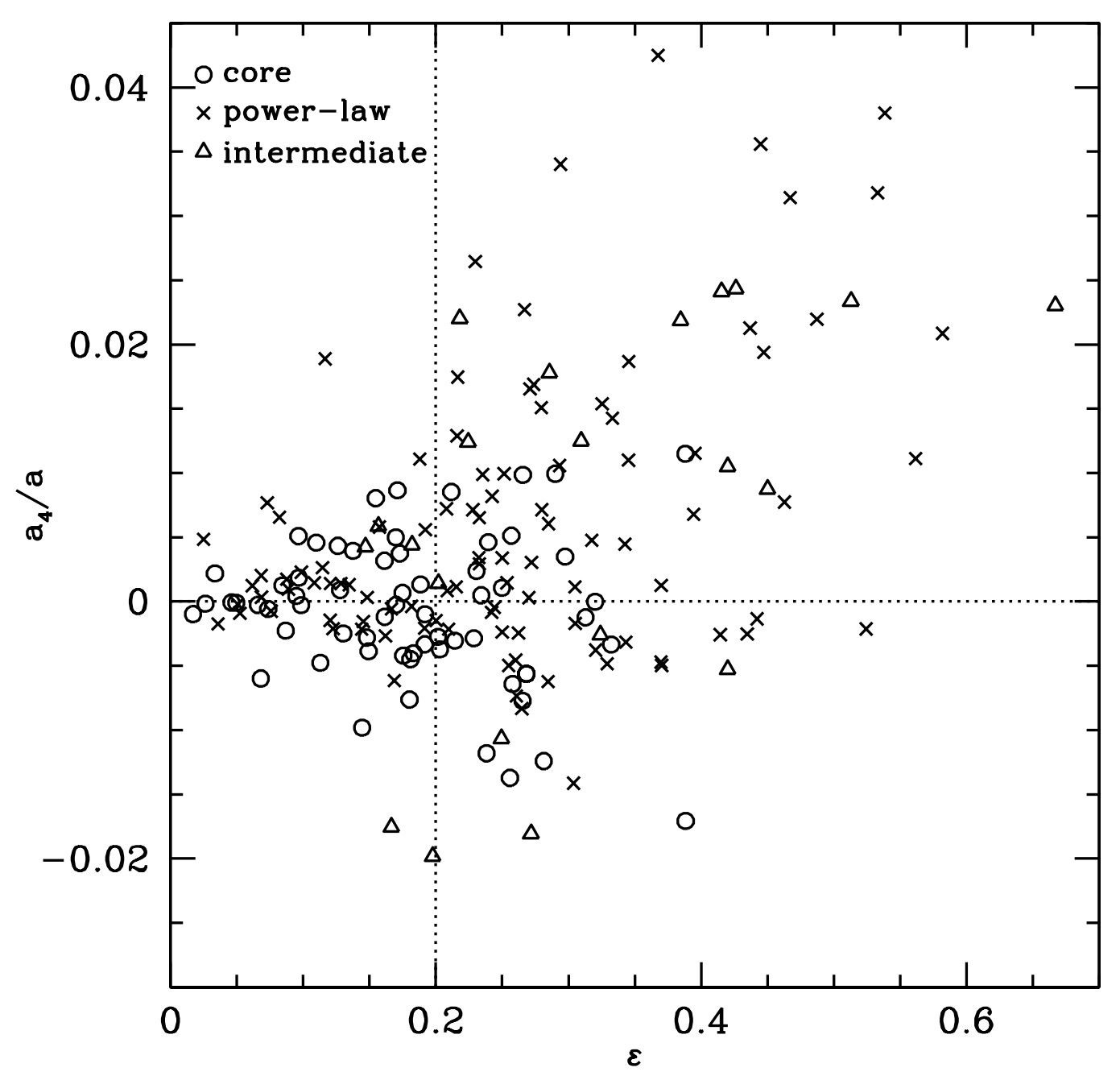

Fig. 6 Isophotal shape parameter $a_{4} / a$ as a function of ellipticity $\epsilon$. The red circles show "core" galaxies, triangles are "intermediate" galaxies, and blue crosses show "power-law" galaxies. The horizontal dotted line divides disky $\left(a_{4} / a>0\right)$ and boxy $\left(a_{4} / a<0\right)$ galaxies. The vertical dotted line shows galaxies with ellipticity separator of $\epsilon=0.2$.

$a_{4} / a$ versus $\epsilon$ are $r_{s}=0.55,0.38$ and Prob $=3.96 \times 10^{-10}, 3.61 \times 10^{-5}$, respectively. It indicates that the correlation between kinematic property and ellipticity is tighter than that between isophotal shape and ellipticity.

\section{SUMMARY}

In this paper, we study the relations among isophotal shapes, central light profiles and kinematic properties of ETGs based on a compiled sample with 184 objects observed with both HST and SDSS DR8. Our main results are summarized as follows: 
1. There are no obvious relations of isophotal parameter $a_{4} / a$ with the central light profile slope $\gamma^{\prime}$ and the central "cusp radius" $r_{\gamma}$. About $41 \%$ "core" ETGs have disky isophotes, and 35\% "power-law" ETGs are boxy distorted.

2. Our statistical results show that there are only weak correlations between $a_{4} / a$ and the galaxy luminosity $M_{V}$, and between $a_{4} / a$ and the dynamical mass. Nuclear profiles correlate with $M_{V}$ and dynamical mass more tightly. In addition, there is no any correlation between $a_{4} / a$ and the Sérsic index $n$.

3. There are similar correlations between $a_{4} / a$ and ellipticity and between $a_{4} / a$ and the specific angular momentum $\lambda_{R_{e} / 2}$, i.e. $a_{4} / a$ is correlated with ellipticity and $\lambda_{R_{e} / 2}$ for "power-law" ETGs, but no such relations for "core" ETGs. Quite a large fraction of fast rotator ETGs (70\%) have disky isophotes, while the slow rotator ETGs (56\%) tend to be boxy. The most deviated disky galaxies (i.e. with highest $a_{4} / a$ ) are fast rotators and "power-law" ETGs.

Our statistical results support the statement by ATLAS ${ }^{3 \mathrm{D}}$ group that isophotal shape $\left(a_{4} / a\right)$ of ETGs has no simple relation with both global and central properties of ETGs, but there seems to be correlation between $a_{4} / a$ and kinematic property for "power-law" ETG. Considering that galaxy formation is a very complicated process as shown by both observations and simulations, which can lead to different morphologies, isophotal shapes, central light profiles, kinematic and other global physical properties. There indeed exist some trend among some physical parameters, but simple bimodal classifications may be too simplistic. As a caveat, our sample is compiled in a somewhat complicated way, so the numbers quoted in this paper may suffer from some selection effects.

Acknowledgements We thank Drs. Shude Mao and C. G. Shu for advice and helpful discussions. We also thank the anonymous referee for constructive comments. This project is supported by the NSF of China 10973011, 10833006, 11003015, the Chinese Academy of Sciences and NAOC (SM). The Project-sponsored by SRF for ROCS, SEM. Funding for the creation and distribution of the SDSS Archive has been provided by the Alfred P. Sloan Foundation, the Participating Institutions, the National Aeronautics and Space Administration, the National Science Foundation, the U.S. Department of Energy, the Japanese Monbukagakusho, and the Max Planck Society. The SDSS Web site is http://www.sdss3.org/ The SDSS is managed by the Astrophysical Research Consortium (ARC) for the Participating Institutions. The Participating Institutions are The University of Chicago, Fermilab, the Institute for Advanced Study, the Japan Participation Group, The Johns Hopkins University, the Korean Scientist Group, Los Alamos National Laboratory, the Max-Planck-Institute for Astronomy (MPIA), the Max-Planck-Institute for Astrophysics (MPA), New Mexico State University, University of Pittsburgh, Princeton University, the United States Naval Observatory, and the University of Washington.

\section{References}

Bender, R., Döbereiner, S., \& Möllenhoff, C. 1988, A\&AS, 74, 385

Bender, R., Surma, P., Döbereiner, S., Möllenhoff, C., \& Madejsky, R. 1989, A\&A, 217, 35

Bertin, E., \& Arnouts, S. 1996, A\&AS, 117, 393

Blanton, M. R., \& Roweis, S. 2007, AJ, 133, 734

Cappellari, M., Emsellem, E., Bacon, R., et al. 2007, MNRAS, 379, 418

Cappellari, M., Emsellem, E., Krajnović, D., et al. 2011, MNRAS, 416, 1680

Crane, P., Stiavelli, M., King, I. R., et al. 1993, AJ, 106, 1371

de Zeeuw, P. T., Bureau, M., Emsellem, E., et al. 2002, MNRAS, 329, 513

Emsellem, E., Cappellari, M., Krajnović, D., et al. 2007, MNRAS, 379, 401

Emsellem, E., Cappellari, M., Krajnović, D., et al. 2011, MNRAS, 414, 888

Faber, S. M., Tremaine, S., Ajhar, E. A., et al. 1997, AJ, 114, 1771

Ferrarese, L., van den Bosch, F. C., Ford, H. C., Jaffe, W., \& O'Connell, R. W. 1994, AJ, 108, 1598

Hao, C. N., Mao, S., Deng, Z. G., Xia, X. Y., \& Wu, H. 2006, MNRAS, 370, 1339

He, Y. Q., Xia, X. Y., Hao, C. N., et al. 2013, ApJ, 773, 37 
Hopkins, P., Cox, T. J., Dutta, S. N., et al. 2009a, ApJS, 181, 135

Hopkins, P., Lauer T. R., Cox, T. J., Hernquist, L., \& Kormendy J. 2009b, ApJS, 181, 486

Hyde, J. B., Bernardi, M., Sheth, R. K., \& Nichol, R. C. 2008, MNRAS, 391, 1559

Khochfar, S. \& Burkert, A. 2005, MNRAS, 359, 1379

Kormendy, J. 2009a, ASPC, 419, 87

Kormendy, J., Fisher, D. B., Cornell, M. E., \& Bender, R. 2009b, ApJS, 182, 216

Krajnović, D., Emsellem, E., Cappellari, M., et al. 2011, MNRAS, 414, 2923

Krajnović, D., Karick, A. M., Davies, R. L., et al. 2013, MNRAS, 433, 2812

Laine, S., van der Marel, R. P., Lauer T. R., et al. 2003, AJ, 125, 478

Lauer, T. R. 1985, MNRAS, 216, 429

Lauer, T. R., Ajhar, E. A., Byun, Y. I., et al. 1995, AJ, 110, 2622

Lauer, T. R., Faber, S. M., Gebhardt, K., et al. 2005, AJ, 129, 2138

Lauer, T. R., Faber, S. M., Richstone, D., et al. 2007a, ApJ, 662, 808

Lauer, T. R., Gebhardt, K., Faber, S. M., et al. 2007b, ApJ, 664, 226

Lauer, T. R. 2012, ApJ, 759, 64

Liu, F. S., Xia, X. Y., Mao, S., Wu, H., \& Deng, Z. G. 2008, MNRAS, 385, 23

Lupton, R., Gunn, J. E. ., Ivezić, Z., Knapp, G. R., \& Kent, S. 2001, ASPC, 238, 269

Naab, T., \& Burkert, A. 2003, ApJ, 597, 893

Naab, T., \& Trujillo, I. 2006, MNRAS, 369, 625

Peng, C. Y., Ho, L. C., Impey, C. D., \& Rix, H. W. 2002, AJ, 124, 266

Quillen, A. C., Bower, G. A., Stritzinger, M. 2000, ApJS, 128, 85

Ravindranath, S., Ho, L. C., Peng, C. Y., Filippenko, A. V., \& Sargent, W. L. 2001, AJ, 122, 653

Rest, A., van den Bosch, F. C., Jaffe, W., et al. 2001, AJ, 121, 2431

Sérsic, J. L. 1968, Atlas de Galaxies Australes. Observatorio Astronomico, Cordoba

Smith, J. A., Tucker, D. L., Kent, S. et al. 2002, AJ, 123, 2121

von der Linden, A., Best, P. N., Kauffmann, G., \& White, S. D. M. 2007, MNRAS, 379, 867 